\title{
WEAK APPROXIMATION FOR TORI OVER $p$-ADIC FUNCTION FIELDS
}

\author{
DAVID HARARI, CLAUS SCHEIDERER AND TAMÁS SZAMUELY
}

\begin{abstract}
We study local-global questions for Galois cohomology over the function field of a curve defined over a p-adic field, the main focus being weak approximation of rational points. We construct a 9-term Poitou-Tate type exact sequence for tori over a field as above (and also a 12-term sequence for finite modules). Like in the number field case, part of the sequence can then be used to analyze the defect of weak approximation for a torus. We also show that the defect of weak approximation is controlled by a certain subgroup of the third unramified cohomology group of the torus.
\end{abstract}

\section{INTRODUCTION}

This paper is the companion piece to [15], containing investigations concerning local-global questions for tori defined over the function field $K$ of a curve over a finite extension of $\mathbf{Q}_{p}$. As recalled in the introduction of [15], our project has been motivated by the recent awakening of interest in local-global principles for group schemes defined over fields of cohomological dimension strictly greater than 2, as documented in the work of Harbater, Hartmann and Krashen [16] as well as Colliot-Thélène, Parimala and Suresh ([7], [8]).

In [15] two of us are studying the Hasse principle for torsors under tori over a field $K$ as above, the main tool being a global duality theorem. Presently our main concern is weak approximation for tori. As in the classical case over number fields, this necessitates going beyond duality and establishing a Poitou-Tate type exact sequence for tori over $K$. Here is our first main result:

Theorem 0.1. (= Theorem 2.9) Let $k$ be a finite extension of $\mathbf{Q}_{p}$, and $T$ a torus defined over the function field of a smooth proper $k$-curve $X$. There is an exact sequence of topological groups

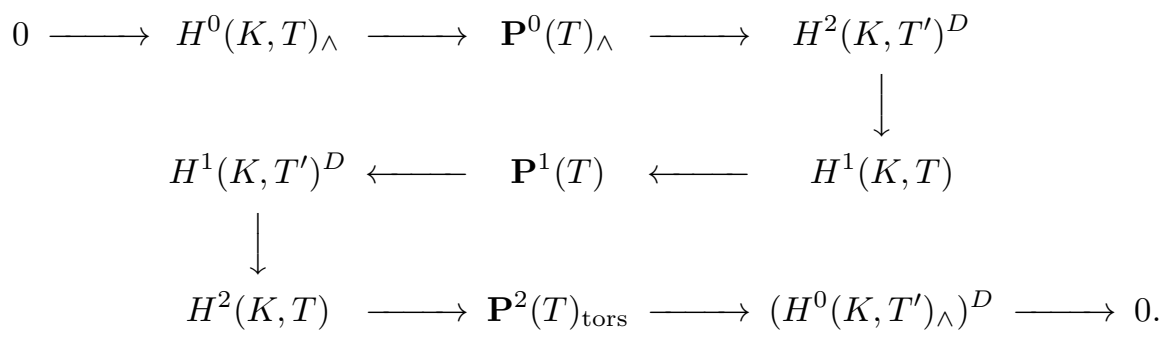

In this sequence, $T^{\prime}$ denotes the dual torus of $T$ (i.e. the torus whose character group is the cocharacter group of $T$ ) and the groups $\mathbf{P}^{i}(T)$ are certain restricted products of local cohomology groups to be defined in Section 2 . Furthermore, the subscript 'tors' stands for the torsion subgroup, the subscript $\wedge$ indicates the inverse limit of $\bmod n$ quotients for all $n>0$, and the superscript $D$ means continuous dual with values in $\mathbf{Q} / \mathbf{Z}$. In Section 2 we shall also construct a 12 -term exact sequence of similar type for finite group schemes over $K$.

Date: January 28, 2014. 
As in the number field case, part of the sequence can be used to analyze the defect of weak approximation for a torus. The question here is whether for $T$ as above the group of points $T(K)$ is dense in the topological product of the groups $T\left(K_{v}\right)$, where $K_{v}$ denotes the completion of $K$ with respect to the discrete valuation associated with a closed point $v \in X$, equipped with its natural topology. The answer is yes when the torus is $K$-rational, but in general there is an obstruction, as shown by the following analogue of a classical result of Voskresenskii ([30; see also [9] and 25]).

Theorem 0.2. (= Theorem $3.3 b)$ ) For $K$ and $X$ as above, denote by $X^{(1)}$ the set of closed points of $X$. For a $K$-torus $T$ let $\overline{T(K)}$ denote the closure of $T(K)$ in the topological product of the $T\left(K_{v}\right)$ for all $v \in X^{(1)}$. There is an exact sequence

$$
0 \rightarrow \overline{T(K)} \rightarrow \prod_{v \in X^{(1)}} T\left(K_{v}\right) \rightarrow \amalg_{\omega}^{2}\left(T^{\prime}\right)^{D} \rightarrow \amalg^{1}(T) \rightarrow 0,
$$

where $\amalg^{1}(T) \subset H^{1}(K, T)$ is the subgroup of classes whose image in $H^{1}\left(K_{v}, T\right)$ is trivial for all $v \in X^{(1)}$, and $\amalg_{\omega}^{2}\left(T^{\prime}\right) \subset H^{2}\left(K, T^{\prime}\right)$ is the subgroup of classes whose image in $H^{2}\left(K_{v}, T^{\prime}\right)$ is trivial for all but finitely many $v$.

Note that, in contrast to the number field case, in our situation the group $\amalg_{\omega}^{2}\left(T^{\prime}\right)$ may be infinite (see Proposition 3.5 below), whereas $\amalg^{1}(T)$ is always finite.

In the last section we reinterpret the above theorem using the reciprocity obstruction to weak approximation first studied by J-L. Colliot-Thélène. In our case this obstruction is defined by means of the subgroup $H_{\mathrm{nr}}^{3}(K(T), \mathbf{Q} / \mathbf{Z}(2)) \subset$ $H^{3}(K(T), \mathbf{Q} / \mathbf{Z}(2))$ of elements having trivial residues for all discrete valuations of $K(T)$ trivial on $K$. (Here $\mathbf{Q} / \mathbf{Z}(2)$ denotes the direct limit of the torsion étale sheaves $\mu_{n}^{\otimes 2}$ for all $n$.) As we shall recall in detail in Section 4, evaluating classes at $K_{v}$-points of $T$ induces a pairing

$$
\prod_{v \in X^{(1)}} T\left(K_{v}\right) \times H_{\mathrm{nr}}^{3}(K(T), \mathbf{Q} / \mathbf{Z}(2)) \rightarrow \mathbf{Q} / \mathbf{Z}
$$

which annihilates the closure of the diagonal image of $T(K)$ on the left. We shall prove:

Theorem 0.3. (= Theorem 4.2) For a $K$-torus $T$ each system $\left(P_{v}\right)$ of local points of $T$ lying in the left kernel in the above pairing is in the closure of the diagonal image of $T(K)$ for the product topology.

In fact, in Theorem 4.2 we shall prove slightly more: we construct a homomorphism $u: \amalg_{\omega}^{2}\left(T^{\prime}\right) \rightarrow H_{\mathrm{nr}}^{3}(K(T), \mathbf{Q} / \mathbf{Z}(2))$ relating the obstruction of Theorem 0.2 to the group $H_{\mathrm{nr}}^{3}(K(T), \mathbf{Q} / \mathbf{Z}(2))$, and show that it is enough to consider the restriction of the pairing to $\operatorname{Im}(u)$ in order to obtain the conclusion of the theorem.

The above theorems were announced, in slightly weaker form, by the second author in seminar lectures given in 2002. The proofs given in the present text were worked out by the first and third authors in 2012-13, in connection with the results of [15. We are very grateful to Jean-Louis Colliot-Thélène and Yves de Cornulier for instructive discussions. Part of this work has been done while the first and third authors were visiting each other's home institutions whose hospitality was greatly appreciated. The first author would also like to thank the Centre Bernoulli (Lausanne) for hospitality and the third author the Hungarian Science Foundation OTKA for partial support under grant No. NK81203.

Notation and conventions. The following conventions will be adopted throughout the article. 
Abelian groups. Given an abelian group $A$, we shall denote by $\bar{A}$ the quotient of $A$ by its maximal divisible subgroup $\operatorname{Div} A$. The $n$-torsion subgroup and (for $\ell$ prime) the $\ell$-primary torsion subgroup of $A$ will be denoted by ${ }_{n} A$ and $A\{\ell\}$, respectively. If $A$ is torsion, it is said to be of cofinite type if for each $n>0$, the $\operatorname{subgroup~}_{n} A$ is finite. For $A$ a topological abelian group the notation $A^{D}$ will stand for the group of continuous homomorphisms $A \rightarrow \mathbf{Q} / \mathbf{Z}$. The functor $A \mapsto A^{D}$ is an anti-equivalence of categories between $\ell$-primary torsion abelian groups of cofinite type (with the discrete topology) and $\mathbf{Z}_{\ell}$-modules of finite type (with the profinite topology).

The notation $A^{\wedge}$ will stand for the profinite completion of $A$, i.e. the inverse limit of its finite quotients. We shall denote by $A_{\wedge}$ the inverse limit of the quotients $A / n A$ for all $n>0$.

Unless otherwise stated, all cohomology groups will be taken with respect to the étale topology.

Tori. For a torus $T$ over a field $F$ we shall denote by $\widehat{T}$ its character module and by $\breve{T}$ its module of cocharacters. These are finitely generated free $\mathbf{Z}$-modules equipped with a Galois action, and moreover $\breve{T}$ is the $\mathbf{Z}$-linear dual of $\widehat{T}$. The dual torus $T^{\prime}$ of $T$ is by definition the $F$-torus with character group $\breve{T}$. The torus $T$ is quasi-trivial if $\widehat{T}$ is a permutation module, i.e. it has a Galois invariant basis. The torus $T$ is flasque if it is split by a finite étale Galois cover of the base with group $G$ and $H^{-1}(H, \widehat{T})=0$ for all subgroups $H \subset G$. It is coflasque if it has a similar property for $H^{1}$ instead of $H^{-1}$. The dual of a flasque torus is coflasque and vice versa. Every torus $T$ has a flasque resolution, i.e. an exact sequence

$$
1 \rightarrow S \rightarrow P \rightarrow T \rightarrow 1
$$

with $P$ a quasi-trivial and $S$ a flasque torus. This holds in fact not just over fields but over arbitrary bases, under very mild assumptions ([10, Theorem 1.3).

Motivic complexes. For $i \geq 0$ and a separated scheme $V$ of finite type over a field $F$ we denote by $z^{i}(X, \bullet)$ Bloch's cycle complex defined in [2]. If $V$ is smooth, the étale motivic complex $\mathbf{Z}(i)$ over $V$ is defined as the complex of sheaves $z^{i}(-, \bullet)[-2 i]$ on the small étale site of $V$. For $i=1$ we have a quasi-isomorphism of complexes of sheaves $\mathbf{Z}(1) \cong \mathbf{G}_{m}[-1]$. The analogously defined Zariski motivic complex will be denoted by $\mathbf{Z}(i)_{\mathrm{Zar}}$. For an abelian group $A$ we denote by $A(i)$ the complex $A \otimes \mathbf{Z}(i)$ and similarly for $\mathbf{Z}(i)_{\text {Zar }}$ (since the terms of $\mathbf{Z}(i)$ are torsion free, this is the same as the derived tensor product). For $m$ invertible in $F$ we have a quasi-isomorphism of complexes of étale sheaves

$$
\mathbf{Z} / m \mathbf{Z}(i) \stackrel{\sim}{\rightarrow} \mu_{m}^{\otimes i}
$$

where $\mu_{m}$ is the étale sheaf of $m$-th roots of unity placed in degree 0 (Geisser-Levine [12, Theorem 1.5). Thus we shall also use the notation $\mathbf{Q} / \mathbf{Z}(i)$ for the direct limit of the sheaves $\mu_{m}^{\otimes i}$ for all $m>0$.

Function fields. Throughout the paper, $k$ will be a finite extension of $\mathbf{Q}_{p}$ for a prime number $p$, and $X$ a smooth proper geometrically integral curve over $k$. The set of all closed points of $X$ will be denoted by $X^{(1)}$, and its function field by $K$. For a closed point $v \in X^{(1)}$ we denote by $\kappa(v)$ its residue field, and by $K_{v}$ the completion of $K$ for the discrete valuation induced by $v$. Then $\kappa(v)$ is also the residue field of the ring of integers $\mathcal{O}_{v}$ and is a finite extension of $k$. Therefore $K_{v}$ is a 2-dimensional local field, i.e. a field complete with respect to a discrete valuation whose residue field is a classical local field. 


\section{DuAlity RESUlts: Summary AND COMPLEMENTS}

In this section we recall the main duality results established in [15] and prove some complements that will be needed for the construction of the Poitou-Tate exact sequence.

Let us start with the local theory. Given a discrete valuation $v$ of the function field $K$ coming from a closed point of the curve $X$, the completion of $K$ at $v$ is a 2-dimensional local field. For such a $K_{v}$ there is a canonical isomorphism

$$
H^{4}\left(K_{v}, \mathbf{Z}(2)\right) \cong \mathbf{Q} / \mathbf{Z}
$$

by 15 , Lemma 2.1 .

If $T$ is a torus over $K$ with dual torus $T^{\prime}$, there is a canonical pairing

$$
T \otimes \mathbf{L} T^{\prime} \rightarrow \mathbf{Z}(2)[2]
$$

in the derived category of étale sheaves over $K_{v}$. It can be constructed using the quasi-isomorphisms

$$
T \cong \breve{T} \otimes^{\mathbf{L}} \mathbf{Z}(1)[1], \quad T^{\prime}=\widehat{T} \otimes^{\mathbf{L}} \mathbf{Z}(1)[1]
$$

(which follow from the quasi-isomorphism $\mathbf{Z}(1)[1] \cong \mathbf{G}_{m}$ ) by putting the pairings $\breve{T} \otimes \widehat{T} \rightarrow \mathbf{Z}$ and $\mathbf{Z}(1)[1] \otimes^{\mathbf{L}} \mathbf{Z}(1)[1] \rightarrow \mathbf{Z}(2)[2]$ together.

We therefore obtain cup-product pairings

$$
H^{i}\left(K_{v}, T\right) \otimes H^{2-i}\left(K_{v}, T^{\prime}\right) \rightarrow H^{4}\left(K_{v}, \mathbf{Z}(2)\right) \cong \mathbf{Q} / \mathbf{Z}
$$

for $i=0,1,2$. Concerning these pairings, we have:

Proposition 1.1. The pairing (41) is a perfect pairing of finite groups for $i=1$. For $i=0$ it becomes a perfect pairing between a profinite and a torsion group, after replacing $H^{0}\left(K_{v}, T\right)$ by its profinite completion.

Proof. See [15], Proposition 2.2.

Recall also (e.g. from [23], I. 2.17) that for a finite $K_{v}$-group scheme $F$ there are perfect pairings of finite groups

$$
H^{i}\left(K_{v}, F\right) \otimes H^{3-i}\left(K_{v}, F^{\prime}\right) \rightarrow H^{3}\left(K_{v}, \mathbf{Q} / \mathbf{Z}(2)\right) \cong \mathbf{Q} / \mathbf{Z}
$$

where $F^{\prime}:=\operatorname{Hom}(F, \mathbf{Q} / \mathbf{Z}(2))$. Note that in the case when $F$ is the $m$-torsion subgroup $_{m} T$ of a $K$-torus $T$ for some $m>0$, we have $F^{\prime} \cong{ }_{m} T^{\prime}$ in view of the chain of isomorphisms

$$
\operatorname{Hom}\left({ }_{m} T, \mu_{m}^{\otimes 2}\right) \cong \operatorname{Hom}\left(\check{T} \otimes \mu_{m}, \mu_{m}^{\otimes 2}\right) \cong \operatorname{Hom}\left(\check{T}, \mu_{m}\right) \cong{ }_{m} T^{\prime} .
$$

We now establish some additional properties of local duality for finite group schemes and for tori.

Proposition 1.2. Assume $F$ is a finite group scheme over $K_{v}$ that extends to a finite and étale group scheme $\mathcal{F}$ over $\operatorname{Spec} \mathcal{O}_{v}$. Then under the pairing

$$
H^{1}\left(K_{v}, F\right) \times H^{2}\left(K_{v}, F^{\prime}\right) \rightarrow \mathbf{Q} / \mathbf{Z}
$$

the subgroups $H^{1}\left(\mathcal{O}_{v}, \mathcal{F}\right) \subset H^{1}\left(K_{v}, F\right)$ and $H^{2}\left(\mathcal{O}_{v}, \mathcal{F}^{\prime}\right) \subset H^{2}\left(K_{v}, F^{\prime}\right)$ are exact annihilators of each other.

In fact, it is not obvious a priori that the maps $H^{1}\left(\mathcal{O}_{v}, \mathcal{F}\right) \rightarrow H^{1}\left(K_{v}, F\right)$ and $H^{2}\left(\mathcal{O}_{v}, \mathcal{F}^{\prime}\right) \rightarrow H^{2}\left(K_{v}, F^{\prime}\right)$ are injective, but this will follow from exact sequences (7) and (8) in the proof below. 
Proof. First of all, the cup-product pairing

$$
H^{i}\left(\mathcal{O}_{v}, \mathcal{F}\right) \times H^{3-i}\left(\mathcal{O}_{v}, \mathcal{F}^{\prime}\right) \rightarrow H^{3}\left(\mathcal{O}_{v}, \mathbf{Q} / \mathbf{Z}(2)\right)
$$

induced by $\mathcal{F} \otimes \mathcal{F}^{\prime} \rightarrow \mathbf{Q} / \mathbf{Z}(2)$ is trivial for all $i$, since

$$
H^{3}\left(\mathcal{O}_{v}, \mathbf{Q} / \mathbf{Z}(2)\right) \cong H^{3}(\kappa(v), \mathbf{Q} / \mathbf{Z}(2))=0,
$$

the residue field $\kappa(v)$ being of cohomological dimension 2 .

Let now $I_{v}$ be the inertia subgroup of the Galois group $G_{v}$ of $K_{v}$, and denote by $G(v)=G_{v} / I_{v}$ the Galois group of the residue field $\kappa(v)$. By assumption, we may identify $F$ with a $G_{v}$-module with trivial $I_{v}$-action, and we have $H^{i}\left(\mathcal{O}_{v}, \mathcal{F}\right)=$ $H^{i}(G(v), F)$ (and likewise for $F^{\prime}$ ). Since $F$ is unramified, the Hochschild-Serre spectral sequence yields a short exact sequence

$$
0 \rightarrow H^{1}(G(v), F) \rightarrow H^{1}\left(G_{v}, F\right) \rightarrow H^{0}\left(G(v), H^{1}(I, F)\right) \rightarrow 0 .
$$

Using moreover the fact that the exact sequence of Galois groups

$$
1 \rightarrow I_{v} \rightarrow G_{v} \rightarrow G(v) \rightarrow 1
$$

splits ([27], §II.4.3), we similarly get a short exact sequence

$$
0 \rightarrow H^{2}\left(G(v), F^{\prime}\right) \rightarrow H^{2}\left(G_{v}, F^{\prime}\right) \rightarrow H^{1}\left(G(v), H^{1}\left(I, F^{\prime}\right)\right) \rightarrow 0 .
$$

On the other hand, the $G(v)$-module $H^{1}(I, F)=\operatorname{Hom}(I, F)$ is isomorphic to $F(-1)$, which is the module of characters of $F^{\prime}$ (and vice versa). Therefore by Tate's local duality theorem for finite Galois modules over $p$-adic fields, the last terms in exact sequences (7) and (8) are dual finite abelian groups. It follows that $H^{1}\left(K_{v}, F\right) / H^{1}\left(\mathcal{O}_{v}, \mathcal{F}\right)$ and $H^{2}\left(K_{v}, F^{\prime}\right) / H^{2}\left(\mathcal{O}_{v}, \mathcal{F}^{\prime}\right)$ are finite of the same cardinality, which shows that $H^{1}\left(\mathcal{O}_{v}, \mathcal{F}\right)$ and $H^{2}\left(\mathcal{O}_{v}, \mathcal{F}^{\prime}\right)$ are indeed exact annihilators.

Proposition 1.3. Let $T$ be a torus over $K_{v}$ that extends to a torus $\mathcal{T}$ over $\operatorname{Spec} \mathcal{O}_{v}$. Then :

a) Under the pairing

$$
H^{1}\left(K_{v}, T\right) \times H^{1}\left(K_{v}, T^{\prime}\right) \rightarrow \mathbf{Q} / \mathbf{Z}
$$

the subgroups $H^{1}\left(\mathcal{O}_{v}, \mathcal{T}\right) \subset H^{1}\left(K_{v}, T\right)$ and $H^{2}\left(\mathcal{O}_{v}, \mathcal{T}^{\prime}\right) \subset H^{2}\left(K_{v}, T^{\prime}\right)$ are exact annihilators of each other.

b) The annihilator of $H^{2}\left(\mathcal{O}_{v}, \mathcal{T}^{\prime}\right)$ under the pairing

$$
H^{0}\left(K_{v}, T\right)^{\wedge} \times H^{2}\left(K_{v}, T^{\prime}\right) \rightarrow \mathbf{Q} / \mathbf{Z}
$$

is $H^{0}\left(\mathcal{O}_{v}, \mathcal{T}\right)^{\wedge}$.

Note that for each $n>0$ the groups $H^{0}\left(K_{v}, T\right) / n$ and their subgroups $H^{0}\left(\mathcal{O}_{v}, \mathcal{T}\right) / n$ are finite. Hence the profinite completions $H^{0}\left(K_{v}, T\right)^{\wedge}$ and $H^{0}\left(\mathcal{O}_{v}, \mathcal{T}\right)^{\wedge}$ equal the ' $n$-adic completions' $H^{0}\left(K_{v}, T\right)_{\wedge}$ and $H^{0}\left(\mathcal{O}_{v}, \mathcal{T}\right)_{\wedge}$, respectively.

Also, as in the previous proposition, the injectivity of the maps $H^{1}\left(\mathcal{O}_{v}, \mathcal{T}\right) \rightarrow$ $H^{1}\left(K_{v}, T\right)$ and $H^{2}\left(\mathcal{O}_{v}, \mathcal{T}^{\prime}\right) \rightarrow H^{2}\left(K_{v}, T^{\prime}\right)$ will be justified in the course of the proof. 
Proof. a) As before, the pairing $H^{1}\left(\mathcal{O}_{v}, \mathcal{T}\right) \times H^{1}\left(\mathcal{O}_{v}, \mathcal{T}^{\prime}\right) \rightarrow \mathbf{Q} / \mathbf{Z}$ is trivial because $H^{3}\left(\mathcal{O}_{v}, \mathbf{Q} / \mathbf{Z}(2)\right)=0$, so it remains to prove that each $t \in H^{1}\left(K_{v}, T\right)$ orthogonal to $H^{1}\left(\mathcal{O}_{v}, \mathcal{T}^{\prime}\right)$ comes from $H^{1}\left(\mathcal{O}_{v}, \mathcal{T}\right)$. By functoriality, for each $n>0$ the image of $t$ in $H^{2}\left(K_{v},{ }_{n} T\right)$ is orthogonal to $H^{1}\left(\mathcal{O}_{v},{ }_{n} T^{\prime}\right)$, hence comes from $H^{2}\left(\mathcal{O}_{v},{ }_{n} \mathcal{T}\right)$ by Proposition 1.2. There is a commutative diagram with exact rows

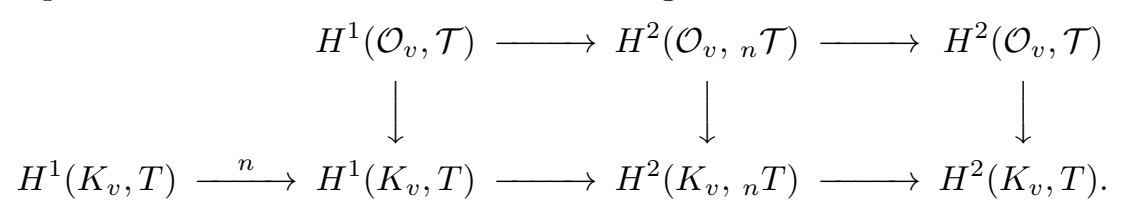

Now observe that the map $H^{2}\left(\mathcal{O}_{v}, \mathcal{T}\right) \rightarrow H^{2}\left(K_{v}, T\right)$ is injective. Indeed, we may identify it with a map $H^{2}\left(G / I, T\left(\bar{K}_{v}\right)^{I}\right) \rightarrow H^{2}\left(G, T\left(\bar{K}_{v}\right)\right)$ in a Hochschild-Serre spectral sequence, where $I$ is the inertia subgroup in $G=\operatorname{Gal}\left(\bar{K}_{v} \mid K_{v}\right)$; this map is injective because $H^{0}\left(G / I, H^{1}(I, T(\bar{K}))\right)=0$ by Hilbert's Theorem 90 as $T$ is split by an unramified extension.

A diagram chase now shows that there exists $t_{0} \in H^{1}\left(\mathcal{O}_{v}, \mathcal{T}\right)$ such that $t-t_{0} \in$ $n H^{1}\left(K_{v}, T\right)$. Since this holds for every $n>0$, the image of $t$ in the finite group $H^{1}\left(K_{v}, T\right) / H^{1}\left(\mathcal{O}_{v}, \mathcal{T}\right)$ is divisible, hence zero.

b) We already know that the pairing is trivial on $H^{0}\left(\mathcal{O}_{v}, \mathcal{T}\right)^{\wedge} \times H^{2}\left(\mathcal{O}_{v}, \mathcal{T}^{\prime}\right)$. Fix $n>0$ and assume $t \in H^{0}\left(K_{v}, T\right) / n$ is orthogonal to ${ }_{n} H^{2}\left(\mathcal{O}_{v}, \mathcal{T}^{\prime}\right)$. By functoriality the image $s$ of $t$ in $H^{1}\left(K_{v},{ }_{n} T\right)$ is orthogonal to $H^{2}\left(\mathcal{O}_{v},{ }_{n} \mathcal{T}^{\prime}\right)$, hence $s \in H^{1}\left(\mathcal{O}_{v},{ }_{n} \mathcal{T}\right)$ by Proposition 1.2 . The exact commutative diagram

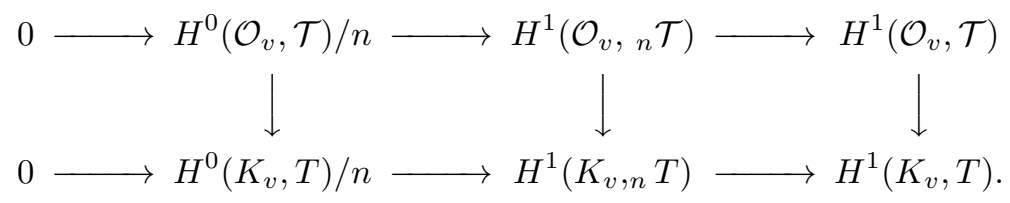

then implies $t \in H^{0}\left(\mathcal{O}_{v}, T\right) / n$ because the right vertical map is injective, again by a Hochschild-Serre argument. This proves the statement.

We conclude this section by recalling the global duality theorems of [15]. Given a torus $T$ over our function field $K$, we set

$$
\amalg^{i}(T):=\operatorname{Ker}\left(H^{i}(K, T) \rightarrow \prod_{v \in X^{(1)}} H^{i}\left(K_{v}, T\right)\right)
$$

for $i \geq 1$. Similar notation will be used for a finite group scheme $F$ over $K$.

Theorem 1.4. Given a K-torus $T$, there is a perfect pairing of finite groups

$$
\amalg^{1}(T) \times \amalg^{2}\left(T^{\prime}\right) \rightarrow \mathbf{Q} / \mathbf{Z} .
$$

Similarly, for a finite group scheme $F$ over $K$ there are perfect pairings of finite groups

for $i=1,2$.

$$
\amalg^{i}(F) \times \amalg^{4-i}\left(F^{\prime}\right) \rightarrow \mathbf{Q} / \mathbf{Z}
$$

Proof. See [15], Theorems 4.1 and 4.4.

\section{Poitou-Tate exact Sequences}

In this section we construct Poitou-Tate type sequences for finite modules and tori over $K$. We start with the case of finite modules. Let $F$ be a finite group scheme over $K$. We know that $F$ extends to a finite étale group scheme $\mathcal{F}$ over a suitable Zariski open $U_{0} \subset X$. For each $i \geq 0$ denote by $\mathbf{P}^{i}(F)$ the topological restricted product of the $H^{i}\left(K_{v}, F\right)$ for all $v \in X^{(1)}$ with respect to the images of 
the maps $H^{i}\left(\mathcal{O}_{v}, \mathcal{F}\right) \rightarrow H^{i}\left(K_{v}, F\right)$ for $v \in U_{0}$ (we shall use the same notation for a $K$-torus instead of a finite module). One sees that $\mathbf{P}^{i}(F)$ does not depend on the choices of $U_{0}$ and of $\mathcal{F}$. We have

$$
\mathbf{P}^{0}(F)=\prod_{v \in X^{(1)}} H^{0}\left(K_{v}, F\right) .
$$

Also,

$$
\mathbf{P}^{3}(F)=\bigoplus_{v \in X^{(1)}} H^{3}\left(K_{v}, F\right)
$$

since

$$
H^{3}\left(\mathcal{O}_{v}, \mathcal{F}\right)=H^{3}\left(\kappa(v), F_{\kappa(v)}\right)=0
$$

for $v \in U_{0}$ because $\kappa(v)$ is a $p$-adic field (hence of cohomological dimension 2). The group $\mathbf{P}^{0}(F)$ is profinite (hence compact) being the product of finite groups, and $\mathbf{P}^{3}(F)$ is discrete as a direct sum of such, but the other two groups are only locally compact.

The following result gives pieces of the Poitou-Tate exact sequence.

Proposition 2.1. For $i=1,2,3$ there are exact sequences

$$
H^{i}(K, F) \rightarrow \mathbf{P}^{i}(F) \stackrel{\theta}{\rightarrow} H^{3-i}\left(K, F^{\prime}\right)^{D}
$$

where the map $\theta$ is defined via the local pairings $\langle,\rangle_{v}$ by the formula

$$
\theta\left(\left(f_{v}\right)\right)\left(f^{\prime}\right)=\sum_{v \in X^{(1)}}\left\langle f_{v}, f_{v}^{\prime}\right\rangle_{v}
$$

for $\left(f_{v}\right) \in \mathbf{P}^{i}(F)$ and $f^{\prime} \in H^{3-i}\left(K, F^{\prime}\right)$.

Note that since for almost all $v$ the module $F$ is unramified at $v$ and moreover $f_{v} \in H^{i}\left(\mathcal{O}_{v}, \mathcal{F}\right)$ and $f_{v}^{\prime} \in H^{3-i}\left(\mathcal{O}_{v}, \mathcal{F}^{\prime}\right)$, the sum in the above formula is actually finite because the pairings (6) over $\mathcal{O}_{v}$ are trivial.

For the proof we need the following lemma (which is in fact valid over an arbitrary base field).

Lemma 2.2. Let $V \subset U$ be nonempty open subsets of $U_{0}$, and let $\alpha \in H^{i}(V, \mathcal{F})$ be such that the localisation $\alpha_{v} \in H^{i}\left(K_{v}, F\right)$ belongs to $H^{i}\left(\mathcal{O}_{v}, \mathcal{F}\right)$ for all $v \in U-V$. Then $\alpha$ is in the image of the restriction map $H^{i}(U, \mathcal{F}) \rightarrow H^{i}(V, \mathcal{F})$.

Proof. The statement follows from the commutative diagram

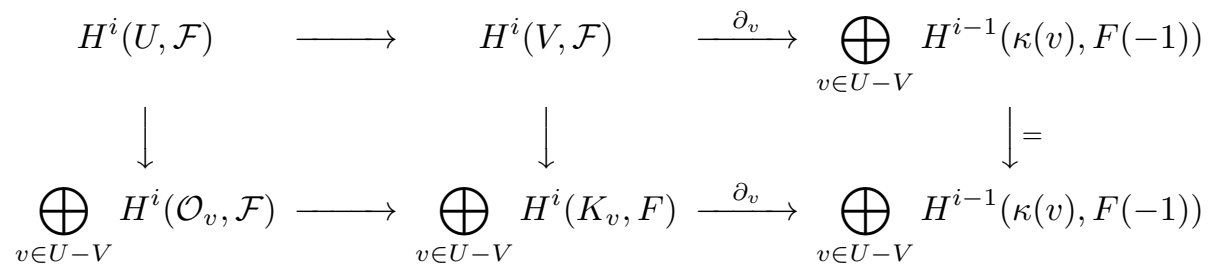

whose exact rows come from localization sequences in étale cohomology.

Proof of Proposition 2.1. Let $U_{0}$ and $\mathcal{F}$ be as above. For every nonempty Zariski open subset $V \subset U_{0}$ there is an exact sequence of finite groups

$$
\cdots \rightarrow H_{c}^{i}(V, \mathcal{F}) \rightarrow H^{i}(V, \mathcal{F}) \rightarrow \bigoplus_{v \in X \backslash V} H^{i}\left(K_{v}, F\right) \rightarrow H_{c}^{i+1}(V, \mathcal{F}) \rightarrow \cdots
$$

constructed as in ([23, Lemma II.2.4), noting that we may replace henselisations at $v$ by completions in view of Greenberg's approximation theorem [13] (see e.g. 
the proof of [14, Lemma 2.7 for a detailed argument). Lemma 2.2 then yields an exact sequence

$$
H^{i}(U, \mathcal{F}) \rightarrow \prod_{v \notin U} H^{i}\left(K_{v}, F\right) \times \prod_{v \in U-V} H^{i}\left(\mathcal{O}_{v}, \mathcal{F}\right) \rightarrow H_{c}^{i+1}(V, \mathcal{F})
$$

for each pair of nonempty open subsets $V \subset U$ contained in $U_{0}$. Moreover, the group $H_{c}^{i+1}(V, \mathcal{F})$ is dual to $H^{3-i}\left(V, \mathcal{F}^{\prime}\right)$ by Artin-Verdier duality for finite modules (see e.g. [11], §5.2). Therefore by taking the inverse limit over $V$ we get an exact sequence

$$
H^{i}(U, \mathcal{F}) \rightarrow \prod_{v \notin U} H^{i}\left(K_{v}, F\right) \times \prod_{v \in U} H^{i}\left(\mathcal{O}_{v}, \mathcal{F}\right) \rightarrow H^{3-i}\left(K, F^{\prime}\right)^{D} .
$$

(In fact, one needs to be a bit careful here since the index set of the inverse limit is uncountable. We have to check that if an element $\left(f_{v}\right)$ of the middle term has trivial image in $H^{3-i}\left(K, F^{\prime}\right)^{D}$, then it comes from $H^{i}(U, \mathcal{F})$. For each nonempty open $V \subset U$ the assumption implies that the image of the truncated element $\left(f_{v}\right)_{v \notin V}$ in $H^{3-i}\left(V, \mathcal{F}^{\prime}\right)^{D}$ is zero, so the inverse image $E_{V}$ of $\left(f_{v}\right)_{v \notin V}$ in $H^{i}(U, \mathcal{F})$ is nonempty by exact sequence (10). On the other hand for $W \subset V$ we obviously have $E_{W} \subset E_{V}$, so the intersection of all subsets $E_{V}$ in the finite set $H^{i}(U, \mathcal{F})$ is nonempty, as required.)

Finally, taking now direct limit over $U$ yields the exact sequence

$$
H^{i}(K, F) \rightarrow \mathbf{P}^{i}(F) \rightarrow H^{3-i}\left(K, F^{\prime}\right)^{D} .
$$

The full statement of the Poitou-Tate sequence is:

Theorem 2.3 (Poitou-Tate sequence for finite modules). Let $F$ be a finite Galois module over $K$. There is a 12-term exact sequence of topological groups:

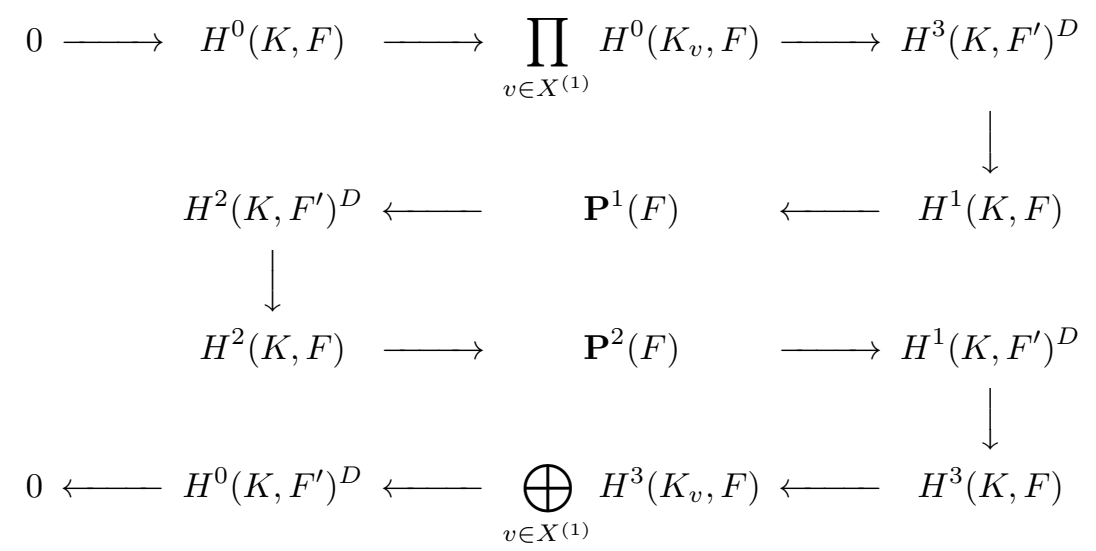

In this statement the groups $H^{i}(K, F)$ and $H^{i}\left(K, F^{\prime}\right)$ are equipped with the discrete topology (hence the dual of $H^{i}\left(K, F^{\prime}\right)$ is compact), and the topology of the restricted products has already been described.

For the proof we need the following general lemma about topological groups which is well known to the experts (whoever they may be) but for which we could not find a reference.

Lemma 2.4. Let

$$
0 \rightarrow A \stackrel{i}{\rightarrow} B \stackrel{p}{\rightarrow} C \rightarrow 0
$$

be an exact sequence of Hausdorff, locally compact and totally disconnected topological abelian groups. Assume that the morphisms $i$ and $p$ are strict. Then the dual 
sequence

$$
0 \rightarrow C^{D} \rightarrow B^{D} \rightarrow A^{D} \rightarrow 0
$$

is exact.

Recall that a continuous homomorphism $f: A \rightarrow B$ of topological groups is said to be strict if $f$ induces an isomorphism between $A / \operatorname{Ker}(f)$ (equipped with the quotient topology) and $\operatorname{Im}(f)$ (equipped with the subspace topology induced by $B)$.

Proof. The injectivity of the map $C^{D} \rightarrow B^{D}$ is obvious. Since $i$ is strict, we can identify $A$ with its image in $B$, equipped with the subspace topology. A continuous homomorphism $B \rightarrow \mathbf{Q} / \mathbf{Z}$ that is trivial on $A$ factors through the topological quotient $B / A$. As $p$ is strict, it thus induces a continuous homomorphism $C \rightarrow \mathbf{Q} / \mathbf{Z}$. This shows the exactness of the dual sequence in the middle, so it remains to justify the surjectivity of the map $B^{D} \rightarrow A^{D}$, which we now do following an argument kindly explained to us by Yves de Cornulier.

Our task is to extend a continuous homomorphism $f: A \rightarrow \mathbf{Q} / \mathbf{Z}$ to a continuous homomorphism $B \rightarrow \mathbf{Q} / \mathbf{Z}$. This is easy if $A$ is open in $B$ : we may extend $f$ to a homomorphism $g: B \rightarrow \mathbf{Q} / \mathbf{Z}$ because $\mathbf{Q} / \mathbf{Z}$ is divisible, and $g$ is automatically continuous because it is continuous in a neighbourhood of 0 by openness of $A$.

To reduce to the above special case, note first that by replacing $A$ and $B$ by their quotients modulo $\operatorname{Ker}(f)$ we may assume that $f$ is injective. This then implies that $A$ has the discrete topology because $\operatorname{Ker}(f)=\{0\}$ is then an open subgroup of $A$, the group $\mathbf{Q} / \mathbf{Z}$ being discrete. Now the assumption that $B$ is locally compact and totally disconnected implies by ([18, Theorem 7.7) that there is a basis of neighborhoods of 0 in $B$ consisting of open subgroups. As $A$ is discrete, we therefore find an open subgroup $U$ of $B$ such that $U \cap A=\{0\}$. Consider the union in $B$ of all open cosets $a+U$ for $a \in A$, and denote it by $A+U$. It is an open subgroup of $B$, and there is a well-defined projection homomorphism $p_{1}: A+U \rightarrow A$ since $U \cap A=\{0\}$. The map $p_{1}$ is also continuous, the preimage of an open subset $V \subset A$ being the union of the open cosets $v+U$ for $v \in V$. Therefore the composite map $f \circ p_{1}: A+U \rightarrow \mathbf{Q} / \mathbf{Z}$ is a continuous homomorphism extending $f$. Replacing $A$ by $A+U$ we reduce to the open case treated above.

Proof of Theorem 2.3. Exactness of the two middle rows follows from Proposition 2.1. That of the third is also a consequence, once we note the surjectivity of the map

$$
\mathbf{P}^{3}(F)=\bigoplus_{v \in X^{(1)}} H^{3}\left(K_{v}, F\right) \rightarrow H^{0}\left(K, F^{\prime}\right)^{D}
$$

This follows from the injectivity of the dual maps $H^{0}\left(K, F^{\prime}\right) \rightarrow H^{0}\left(K_{v}, F^{\prime}\right)$ via the local duality theorem for finite modules. The first row becomes the dual of the last one if we exchange $F$ and $F^{\prime}$, so it is exact as well, the last row being an exact sequence of discrete torsion groups. The vertical maps are defined using Theorem 1.4. It remains to show that the dual of the exact sequence

$$
0 \rightarrow \amalg^{i}\left(F^{\prime}\right) \rightarrow H^{i}\left(K, F^{\prime}\right) \rightarrow \mathbf{P}^{i}\left(F^{\prime}\right)
$$

is still exact for $i=1,2,3$. Since local duality (5) induces a perfect duality of the locally compact groups $\mathbf{P}^{i}(F)$ and $\mathbf{P}^{3-i}\left(F^{\prime}\right)$ (this uses Proposition 1.2 for $i=1,2$ ), we can then conclude the exactness of

$$
\mathbf{P}^{3-i}(F) \rightarrow H^{i}\left(K, F^{\prime}\right)^{D} \rightarrow \amalg^{4-i}(F) \rightarrow 0 .
$$

To do so, we apply Lemma 2.4, so we only have to prove that the morphism $H^{i}\left(K, F^{\prime}\right) \rightarrow \mathbf{P}^{i}\left(F^{\prime}\right)$ is strict. It will suffice to show that its image is discrete. For each open subset $U \subset U_{0}$ (where $U_{0}$ is chosen such that $F^{\prime}$ extends to a finite 
and étale group scheme $\mathcal{F}^{\prime}$ over $\left.U_{0}\right)$, the group $H^{i}\left(U, \mathcal{F}^{\prime}\right)$ is finite. Since each element of the image of $H^{i}\left(K, F^{\prime}\right)$ in $\prod_{v \notin U} H^{i}\left(K_{v}, F^{\prime}\right) \times \prod_{v \in U} H^{i}\left(\mathcal{O}_{v}, \mathcal{F}^{\prime}\right)$ comes from $H^{i}\left(U, \mathcal{F}^{\prime}\right)$ by Lemma 2.2, we are done.

We now turn to tori. The following proposition is similar to Proposition 2.1

Proposition 2.5. Let $T$ be a $K$-torus. There are exact sequences of topological groups

$$
H^{1}(K, T) \rightarrow \mathbf{P}^{1}(T) \rightarrow H^{1}\left(K, T^{\prime}\right)^{D}
$$

and

$$
H^{2}(K, T) \rightarrow \mathbf{P}^{2}(T)_{\text {tors }} \rightarrow\left(H^{0}\left(K, T^{\prime}\right)_{\wedge}\right)^{D} \rightarrow 0 .
$$

Here $\mathbf{P}^{1}(T)$ is a topological restricted product of finite discrete groups. The topology on $\mathbf{P}^{2}(T)_{\text {tors }}$ is defined as follows. For each $n>0$ the group ${ }_{n} \mathbf{P}^{2}(T)$ (restricted product of the ${ }_{n} H^{2}\left(K_{v}, T\right)$ with respect to the ${ }_{n} H^{2}\left(\mathcal{O}_{v}, \mathcal{T}\right)$ ) is equipped with the restricted product topology associated with the discrete topology on each ${ }_{n} H^{2}\left(K_{v}, T\right)$. Their direct limit $\mathbf{P}^{2}(T)$ tors is equipped with the direct limit topology and not by the subspace topology induced by $\mathbf{P}^{2}(T)$.

Before starting the proof, we have to recall from [15] some properties of the cohomology of tori over open subcurves of $X$.

Lemma 2.6. Let $U \subset X$ be a nonempty open subset such that $T$ extends to a torus $\mathcal{T}$ over $U$.

(1) For each $i \geq 1$ the group $H^{i}(U, \mathcal{T})_{\text {tors }}$ is of cofinite type, and so is the compact support cohomology group $H_{c}^{i}(U, \mathcal{T})_{\text {tors }}$ for $i \geq 2$.

(2) For $U$ sufficiently small the group $H_{c}^{2}(U, \mathcal{T})\{\ell\}$ is finite.

(3) There is a canonical pairing

$$
H^{i}\left(U, \mathcal{T}^{\prime}\right) \otimes H_{c}^{3-i}(U, \mathcal{T}) \rightarrow \mathbf{Q} / \mathbf{Z}
$$

inducing a perfect pairing of finite groups

$$
\overline{H^{1}\left(U, \mathcal{T}^{\prime}\right)\{\ell\}} \times \overline{H_{c}^{2}(U, \mathcal{T})}\{\ell\} \rightarrow \mathbf{Q}_{\ell} / \mathbf{Z}_{\ell}
$$

for each prime number $\ell$.

Proof. The first statement is ([15, Proposition 3.4 (1)). To prove (2), note first that $H_{c}^{2}(U, \mathcal{T})$ is a torsion group ([15, Corollary 3.3). Propositions 4.2 and 3.6 of 15] imply that there is a well-defined map

$$
\bigoplus_{v \in X^{(1)}} H^{1}\left(K_{v}, T\right) \rightarrow H_{c}^{2}(U, \mathcal{T})
$$

whose cokernel has finite $\ell$-primary part for $U$ sufficiently small. Since the groups $H^{1}\left(K_{v}, T\right)$ have bounded exponent by Hilbert's Theorem 90 and a restrictioncorestriction argument, the finiteness of $H_{c}^{2}(U, \mathcal{T})\{\ell\}$ follows from statement (1). Finally, the last statement follows from the Artin-Verdier style result of ([15], Theorem 1.3), noting that for an $\ell$-primary torsion group $A$ of cofinite type the quotient $\bar{A}$ modulo the maximal divisible subgroup is isomorphic to the inverse limit $\lim _{\longleftarrow} A / \ell^{m} A$.

Proof of Proposition 2.5. Extend $T, T^{\prime}$ to tori $\mathcal{T}, \mathcal{T}^{\prime}$ over a nonempty Zariski affine open subset $U_{0} \subset X$. We first prove the first statement along the lines of 
Proposition 2.1. The $K$-torus $T$ is split by an extension of degree $m>0$. Given nonempty open subsets $V \subset U \subset U_{0}$, there is an exact sequence

$$
H^{1}(U, \mathcal{T}) \rightarrow \prod_{v \notin U} H^{1}\left(K_{v}, T\right) \times \prod_{v \in U-V} H^{1}\left(\mathcal{O}_{v}, \mathcal{T}\right) \rightarrow H_{c}^{2}(V, \mathcal{T})
$$

obtained similarly as (10), except that we use Harder's lemma ([17, Lemma 4.1.3) instead of Lemma 2.2. The $K$-torus $T$ is split by some field extension of degree $m>0$, so the middle term has exponent dividing $m$ by Hilbert's Theorem 90 . We therefore obtain an exact sequence

$$
H^{1}(U, \mathcal{T}) / m \rightarrow \prod_{v \notin U} H^{1}\left(K_{v}, T\right) \times \prod_{v \in U-V} H^{1}\left(\mathcal{O}_{v}, \mathcal{T}\right) \rightarrow{ }_{m} H_{c}^{2}(V, \mathcal{T})
$$

whose terms are finite by Proposition 2.6(1). We know by Lemma 2.6 (2) that for $V$ sufficiently small the group $H_{c}^{2}(V, \mathcal{T})\{\ell\}$ is finite, and therefore its maximal divisible subgroup is trivial. Lemma 2.6 (3) then implies that the map $H_{c}^{2}(V, \mathcal{T})\{\ell\} \rightarrow$ $H^{1}\left(V, \mathcal{T}^{\prime}\right)\{\ell\}^{D}$ is injective, whence an exact sequence of finite groups

$$
H^{1}(U, \mathcal{T}) / m \rightarrow \prod_{v \notin U} H^{1}\left(K_{v}, T\right) \times \prod_{v \in U-V} H^{1}\left(\mathcal{O}_{v}, \mathcal{T}\right) \rightarrow \prod_{\ell \mid m} H^{1}\left(V, \mathcal{T}^{\prime}\right)\{\ell\}^{D} .
$$

Taking the inverse limit over $V \subset U$ as in the proof of Proposition 2.1] and noting that $H^{1}\left(K, T^{\prime}\right)$ is also of exponent dividing $m$, we get an exact sequence

$$
H^{1}(U, \mathcal{T}) / m \rightarrow \prod_{v \notin U} H^{1}\left(K_{v}, T\right) \times \prod_{v \in U} H^{1}\left(\mathcal{O}_{v}, \mathcal{T}\right) \rightarrow H^{1}\left(K, T^{\prime}\right)^{D} .
$$

Replacing $H^{1}(U, \mathcal{T}) / m$ by $H^{1}(U, \mathcal{T})$ and taking the direct limit along the subsets $U$ we then obtain exact sequence (11).

To prove the second statement, we fix an integer $n>0$ and start with the exact sequence

$$
H^{2}\left(K,{ }_{n} T\right) \rightarrow \mathbf{P}^{2}\left({ }_{n} T\right) \rightarrow H^{1}\left(K,{ }_{n} T^{\prime}\right)^{D} \rightarrow H^{3}\left(K,{ }_{n} T\right)
$$

given by Theorem 2.3 . Consider the commutative diagram with exact first row

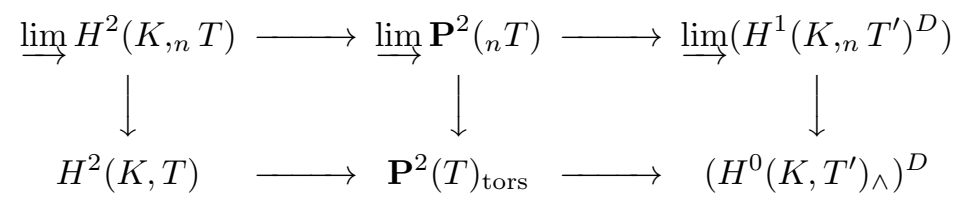

where the dual of the third vertical map comes from the exact sequence

$$
0 \rightarrow H^{0}\left(K, T^{\prime}\right) / n \rightarrow H^{1}\left(K,{ }_{n} T^{\prime}\right) \rightarrow{ }_{n} H^{1}\left(K, T^{\prime}\right) \rightarrow 0 .
$$

The map in question is in fact an isomorphism because the Tate module of $H^{1}\left(K, T^{\prime}\right)$ is zero, the group $H^{1}\left(K, T^{\prime}\right)$ being of finite exponent. Similarly, the first vertical map is an isomorphism because $H^{1}(K, T)$ is torsion and hence $H^{1}(K, T) \otimes \mathbf{Q} / \mathbf{Z}=0$. Since the maps $H^{2}\left(K_{v},{ }_{n} T\right) \rightarrow{ }_{n} H^{2}\left(K_{v}, T\right)$ and $H^{2}\left(\mathcal{O}_{v},{ }_{n} \mathcal{T}\right) \rightarrow{ }_{n} H^{2}\left(\mathcal{O}_{v}, \mathcal{T}\right)$ are onto (the latter for $v \in U_{0}$ ), the middle vertical map is also onto. A diagram chase then yields exact sequence (12) except for the zero on the right. By the diagram and by the exact sequence of Theorem 2.3 this will follow if we prove that the direct limit over $n$ of the groups $H^{3}\left(K,{ }_{n} T\right)$ is trivial. Note first that this direct limit is $H^{3}(K, T)$ because $H^{2}(K, T) \otimes \mathbf{Q} / \mathbf{Z}=0$. But $H^{3}(K, T)$ is the direct limit of the groups $H^{3}(V, \mathcal{T})$ for $V \subset U_{0}$ and the latter groups are trivial by ([26], Corollary 4.10).

We shall also need the following consequence of local duality. 
Lemma 2.7. The local duality theorem induces a perfect duality between the locally compact groups $\mathbf{P}^{1}(T)$ and $\mathbf{P}^{1}\left(T^{\prime}\right)$. It also induces an isomorphism

$$
\left(\mathbf{P}^{2}(T)_{\text {tors }}\right)^{D} \simeq \mathbf{P}^{0}\left(T^{\prime}\right)_{\wedge}
$$

Proof. The first assertion is an immediate consequence of Prop. $1.3 a$ ). The second one follows from part $b$ ) of the proposition, the definition of the topology on $\mathbf{P}^{2}(T)_{\text {tors }}$ as well as the canonical isomorphism between $\mathbf{P}^{0}(T) / n$ and the restricted product of the $H^{0}\left(K_{v}, T\right) / n$ for each $n>0$. To see that the natural surjections $\mathbf{P}^{0}(T) / n \rightarrow H^{0}\left(K_{v}, T\right) / n$ indeed induce an isomorphism with the restricted product it suffices to show the injectivity of the maps $H^{0}\left(\mathcal{O}_{v}, \mathcal{T}\right) / n \rightarrow H^{0}\left(K_{v}, T\right) / n$. This in turn follows from the injectivity of the maps $H^{1}\left(\mathcal{O}_{v},{ }_{n} \mathcal{T}\right) \rightarrow H^{1}\left(K_{v},{ }_{n} T\right)$ in view of the Kummer sequence.

Finally, we shall use an analogue of Lemma 2.2 (again valid over an arbitrary base field).

Lemma 2.8. Assume $T$ extends to a torus $\mathcal{T}$ over a nonempty Zariski open subset $U \subset X$, let $V \subset U$ be a nonempty open subset, and $i \geq 2$ an integer. If $\alpha \in H^{i}(V, T)$ is such that its restriction $\alpha_{v} \in H^{i}\left(K_{v}, T\right)$ lies in $H^{i}\left(\mathcal{O}_{v}, \mathcal{T}\right)$ for each $v \in U-V$, then $\alpha$ is in the image of $H^{i}(U, \mathcal{T})$.

Proof. Lift $\alpha$ to some $\alpha_{n} \in H^{i}\left(V,{ }_{n} T\right)$ for $n>0$. For $v \in U-V$ the image of the restriction $\alpha_{n, v} \in H^{i}\left(K_{v},{ }_{n} T\right)$ in $H^{i}\left(K_{v}, T\right)$ comes from an element in $H^{i}\left(\mathcal{O}_{v}, \mathcal{T}\right)$. We have a commutative diagram with injective vertical maps:

$$
\begin{aligned}
& \underset{n}{\lim _{n}} H^{i}\left(\mathcal{O}_{v},{ }_{n} \mathcal{T}\right) \stackrel{\simeq}{\longrightarrow} H^{i}\left(\mathcal{O}_{v}, \mathcal{T}\right) \\
& \downarrow \\
& \underset{n}{\lim } H^{i}\left(K_{v},{ }_{n} T\right) \stackrel{\simeq}{\longrightarrow} H^{i}\left(K_{v}, T\right) .
\end{aligned}
$$

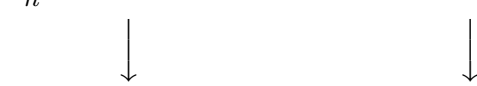

The horizontal maps are isomorphisms because $H^{i-1}\left(\mathcal{O}_{v}, \mathcal{T}\right) \otimes \mathbf{Q} / \mathbf{Z}=0$ for $i \geq 2$. Since there are only finitely many places $v$ in $U-V$, there exists a multiple $m$ of $n$ such that the image $\alpha_{m}$ of $\alpha_{n}$ in $H^{i}(V, m \mathcal{T})$ has the following property: for each $v \in U-V$, the restriction $\alpha_{m, v}$ belongs to $H^{i}\left(\mathcal{O}_{v}, m \mathcal{T}\right)$. By Lemma 2.2 this implies that $\alpha_{m}$ is in the image of $H^{i}(U, m \mathcal{T})$, hence $\alpha$ is in the image of $H^{i}(U, \mathcal{T})$.

We now arrive at

Theorem 2.9 (Poitou-Tate sequence for tori). Let $T$ be a K-torus. There is an exact sequence of topological groups:

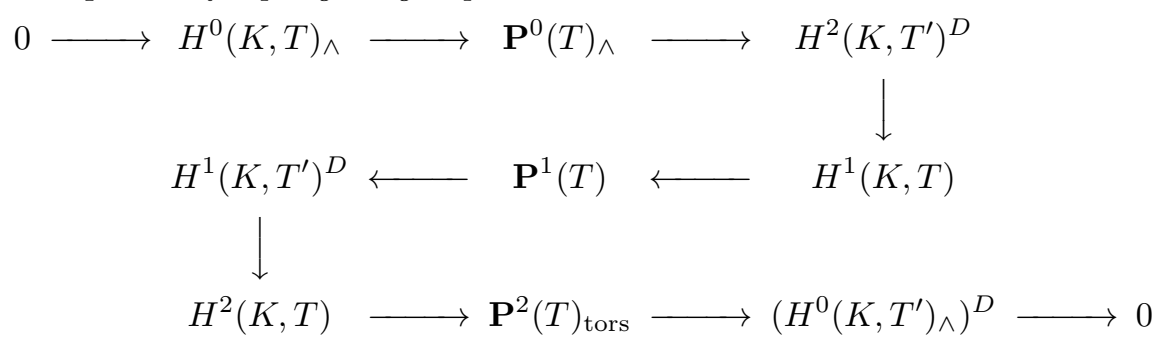

Proof. Exactness of the second and third rows follows from Proposition 2.5. The vertical maps are defined using Theorem 1.4. The exactness of

$$
\mathbf{P}^{1}(T) \rightarrow H^{1}\left(K, T^{\prime}\right)^{D} \rightarrow \amalg^{2}(T) \rightarrow 0
$$

is proven as in Theorem 2.3. replacing the use of Proposition 1.2 by that of Proposition 1.3 and the finiteness of $H^{1}\left(U, \mathcal{F}^{\prime}\right)$ by the finiteness of $H^{1}\left(U, \mathcal{T}^{\prime}\right) / m$, where $T^{\prime}$ is split by an extension of degree $m$. 
To get the exactness of

$$
\mathbf{P}^{0}(T)_{\wedge} \rightarrow H^{2}\left(K, T^{\prime}\right)^{D} \rightarrow \amalg^{1}(T) \rightarrow 0,
$$

we observe that by Lemma 2.7 it is the dual of the exact sequence

$$
0 \rightarrow \amalg^{2}\left(T^{\prime}\right) \rightarrow H^{2}\left(K, T^{\prime}\right) \rightarrow \mathbf{P}^{2}\left(T^{\prime}\right)_{\text {tors }}
$$

To see that the dual remains exact, we need to check, again using Lemma 2.4 that $H^{2}\left(K, T^{\prime}\right) \rightarrow \mathbf{P}^{2}\left(T^{\prime}\right)_{\text {tors }}$ is a strict morphism. Again we show that in fact the image $I$ of $H^{2}\left(K, T^{\prime}\right)$ is discrete in $\mathbf{P}^{2}\left(T^{\prime}\right)_{\text {tors }}$. Fix $n>0$, and let $A_{n}$ be the inverse image in $H^{2}\left(K, T^{\prime}\right)$ of the subgroup $B_{n}$ of $\mathbf{P}^{2}\left(T^{\prime}\right)_{\text {tors }}$ defined by

$$
B_{n}:=\prod_{v \notin U}{ }_{n} H^{2}\left(K_{v}, T^{\prime}\right) \times \prod_{v \in U}{ }_{n} H^{2}\left(\mathcal{O}_{v}, \mathcal{T}^{\prime}\right) .
$$

By Lemma 2.8, the group $A_{n}$ lies in the image of the map $H^{2}\left(U, \mathcal{T}^{\prime}\right) \rightarrow H^{2}\left(K, T^{\prime}\right)$ (lift first elements of $A_{n}$ to $H^{2}\left(V, \mathcal{T}^{\prime}\right)$ for $V$ sufficiently small). Therefore $A_{n}$ is a torsion group of cofinite type by Proposition 2.6 (1), which implies that $A_{n} / n$ is finite. In particular the image of $A_{n}$ in the $n$-torsion group $B_{n}$ is finite. Since this image is $I \cap B_{n}$, we obtain that $I$ is discrete in $\mathbf{P}^{2}\left(T^{\prime}\right)_{\text {tors }}$ by definition of the topology on $\mathbf{P}^{2}\left(T^{\prime}\right)_{\text {tors }}$.

Finally, the exactness of the first row is obtained by dualizing the last one (after exchanging $T$ and $T^{\prime}$ ), once we have observed that for each $n>0$ the bidual of the discrete torsion group $H^{0}\left(K, T^{\prime}\right) / n$ is itself. The dual of the last row remains exact because by definition of the topology on $\mathbf{P}^{2}(T)$ tors and the equality $\left(H^{0}\left(K, T^{\prime}\right)_{\wedge}\right)^{D}=\underset{n}{\lim }\left(H^{0}\left(K, T^{\prime}\right) / n\right)^{D}$ the surjection $\mathbf{P}^{2}(T)_{\text {tors }} \rightarrow\left(H^{0}\left(K, T^{\prime}\right)_{\wedge}\right)^{D}$ is strict.

Remark 2.10. The above sequence looks like the Poitou-Tate exact sequence for a finite module (or a torus) over a number field. But there is an important difference: the group $\mathbf{P}^{2}(T)_{\text {tors }}$ is not discrete (neither is its quotient by the image of $\left.H^{2}(K, T)\right)$, and its dual is not compact.

\section{Obstruction to WeAK approximation FOR tORI}

In this section we address weak approximation questions for tori. First some notation: for a finite set of places $S \subset X^{(1)}$ and a positive integer $i$ we define

$$
\amalg_{S}^{i}(F):=\operatorname{Ker}\left[H^{i}(K, F) \rightarrow \prod_{v \notin S} H^{i}\left(K_{v}, F\right)\right]
$$

for every finite group scheme $F$ over $K$. We also define $\amalg_{\omega}^{i}(F) \subset H^{i}(K, F)$ as the subgroup of elements whose restriction to $H^{i}\left(K_{v}, F\right)$ is zero for all but finitely many $v \in X^{(1)}$. Thus $\amalg_{\omega}^{i}(F)$ is the direct limit of the $\amalg_{S}^{i}(F)$ over all finite $S \subset X^{(1)}$. Similar notation will be used for tori.

We start with the following consequence of Proposition 2.1.

Lemma 3.1. Let $S \subset X^{(1)}$ be a finite set of places, and let $F$ be a finite group scheme over $K$. For $i=1,2$ there are exact sequences

$$
H^{i}(K, F) \rightarrow \prod_{v \in S} H^{i}\left(K_{v}, F\right) \rightarrow \amalg_{S}^{3-i}\left(F^{\prime}\right)^{D}
$$

where the second map is given by

$$
\left(f_{v}\right) \mapsto\left(g \mapsto \sum_{v \in S}\left\langle f_{v}, g_{v}\right\rangle_{v}\right) \quad \text { for } \quad\left(f_{v}\right) \in \prod_{v \in S} H^{i}\left(K_{v}, F\right), g \in \amalg_{S}^{3-i}\left(F^{\prime}\right) .
$$


Proof. Proposition 2.1 applied to $F^{\prime}$ implies an exact sequence

$$
\amalg_{S}^{i}\left(F^{\prime}\right) \rightarrow \prod_{v \in S} H^{i}\left(K_{v}, F^{\prime}\right) \rightarrow H^{3-i}(K, F)^{D}
$$

because an element in the middle group can be completed by 0 's to yield an element of $\mathbf{P}^{i}\left(F^{\prime}\right)$. Now the result is obtained by dualizing, replacing $i$ by $3-i$ and applying local duality (5).

Next comes the key finiteness lemma. Its first part generalizes Remark 3.5 of [15].

Lemma 3.2. Let $T$ be a $K$-torus, and $S \subset X^{(1)}$ a finite set of places.

a) If $T$ is quasi-trivial, then $\amalg_{S}^{2}(T)=\amalg_{\omega}^{2}(T)=0$.

b) In general the group $\amalg_{\omega}^{2}(T)$ is of finite exponent and $\amalg_{S}^{2}(T)$ is finite.

Proof. a) (after J-L. Colliot-Thélène). Since $\amalg_{\omega}^{2}(T)$ is the union of all $\amalg_{S}^{2}(T)$ for $S$ finite, we only have to prove the statement about $\amalg_{S}^{2}(T)$. By Shapiro's lemma it is sufficient to deal with the case $T=\mathbf{G}_{m}$. Each element $\alpha \in \amalg_{S}^{2}\left(\mathbf{G}_{m}\right)$ comes from an element $\alpha_{U} \in \operatorname{Br} U$ for some open $U \subset X$ whose restriction to $\operatorname{Br} K_{v}$ is 0 for all $v \in U$. It is sufficient to prove that $\alpha_{U} \in \operatorname{Br} X$. Indeed, this will then imply $\alpha_{U} \in \operatorname{Br} \mathcal{O}_{X, v}$ for all $v \in X^{(1)}$, where $\mathcal{O}_{X, v}$ is the local ring of $X$ at the closed point $v$ whose completion is the ring of integers $\mathcal{O}_{v}$ of $K_{v}$. Since the restriction map $\operatorname{Br} \mathcal{O}_{v} \rightarrow \operatorname{Br} K_{v}$ is injective, we conclude that $\alpha_{U}$ maps to 0 already in $\operatorname{Br} \mathcal{O}_{v}$. Composing with the isomorphism $\operatorname{Br} \mathcal{O}_{v} \cong \operatorname{Br} \kappa(v)$ we conclude that $\alpha_{U}$ maps to 0 by the 'evaluation at $v$ ' map $\operatorname{Br} X \rightarrow \operatorname{Br} \kappa(v)$ induced by $v$. Therefore $\alpha_{U}$ is orthogonal to $\operatorname{Pic} X$ for Lichtenbaum's duality pairing $\operatorname{Br} X \times \operatorname{Pic} X \rightarrow \mathbf{Q} / \mathbf{Z}$ which is defined in [22] by a sum of evaluation maps, from which we conclude $\alpha_{U}=0$.

Pick $v \in X-U$ and set $k^{\prime}:=\kappa(v)$. We have to show that the residue $\chi \in$ $H^{1}\left(k^{\prime}, \mathbf{Q} / \mathbf{Z}\right)$ of $\alpha$ at $v$ is zero. Fix a positive integer $m$ such that $\chi \in H^{1}\left(k^{\prime}, \mathbf{Z} / m\right)$. Since $H^{1}\left(k^{\prime}, \mathbf{Z} / m\right) \cong H^{1}\left(\mathcal{O}_{X, v}^{h}, \mathbf{Z} / m\right)$, we may lift $\chi \in H^{1}\left(k^{\prime}, \mathbf{Z} / m\right)$ to an element $\tilde{\chi} \in H^{1}(\widetilde{V}, \mathbf{Z} / m)$ for an étale neighbourhood $f: \widetilde{V} \rightarrow X$ of $v$ containing a point $w$ with $f(w)=v$ and $\kappa(w)=k^{\prime}$. We may further assume $\widetilde{V}$ to be integral and write $\widetilde{K}$ for its function field. Let $\pi$ be a uniformizing parameter of the local $\operatorname{ring} \mathcal{O}_{\widetilde{V}, w}$. We define an element $\beta$ of $\operatorname{Br} \widetilde{K}$ by

$$
\beta=f^{*}(\alpha)-(\pi \cup \tilde{\chi}),
$$

where $\pi$ is viewed in $\widetilde{K}^{\times} / \widetilde{K}^{\times m}=H^{1}\left(\widetilde{K}, \mu_{m}\right)$. By a standard residue computation, the residue of $\beta$ at $w$ is zero, and after shrinking $\widetilde{V}$ if necessary we can assume that $\beta \in \operatorname{Br} \widetilde{V}$. Since $f$ is étale, by the implicit function theorem it induces a homeomorphism for the $p$-adic topology from an open neighborhood $\widetilde{\Omega}$ of $w$ onto an open neighborhood $\Omega$ of $v$. We may further assume $\Omega-\{v\} \subset U\left(k^{\prime}\right)$. Since $\beta \in \operatorname{Br} \widetilde{V}$, we can assume (shrinking $\widetilde{\Omega}$ if necessary) that the evaluation map

$$
\widetilde{\Omega} \rightarrow \operatorname{Br} k^{\prime}, \quad \widetilde{P} \mapsto \beta(\widetilde{P})
$$

is constant (see for example [3], Lemma 6.2). Then, using the assumption $\alpha_{U, u}=0$ for $u \in U$, we see that the evaluation map

$$
\widetilde{\Omega}-\{w\} \rightarrow \operatorname{Br} k^{\prime}, \quad \widetilde{P} \mapsto f^{*}(\alpha)(\widetilde{P})
$$

is identically 0 , so the map

$$
\widetilde{\Omega}-\{w\} \rightarrow \operatorname{Br} k^{\prime}, \quad \widetilde{P} \mapsto(\pi \cup \tilde{\chi})(\widetilde{P})
$$

is constant. 
Shrinking again $\widetilde{V}$ if necessary, we can find a Zariski open subset $W \subset \mathbf{A}_{k^{\prime}}^{1}=$ $\operatorname{Spec}\left(k^{\prime}[t]\right)$ containing 0 and a smooth $k^{\prime}$-morphism $\varphi: \widetilde{V} \rightarrow W$ sending $w$ to 0 such that the pullback of $t \in \mathcal{O}_{W, 0}$ to $\mathcal{O}_{\widetilde{V}, w}$ is $\pi$. Using again the implicit function theorem, we find a $p$-adic neighborhood $\Omega_{1}$ of $0 \in W\left(k^{\prime}\right)$ such that the evaluation map

$$
\Omega_{1}-\{0\} \rightarrow \operatorname{Br} k^{\prime}, \quad P \mapsto(t \cup \chi)(P)=P \cup \chi
$$

is constant. In fact, it is identically 0 because if we take a uniformizing parameter $\pi_{k^{\prime}} \in \mathcal{O}_{k^{\prime}}$ and consider $P=\pi_{k^{\prime}}^{r m}$ for $r$ large enough, then $P \in \Omega_{1}$ and also $P \cup \chi=0$ as $m \chi=0$. Now considering elements of the form $u . \pi_{k^{\prime}}^{n}$ for large $n$ with some unit $u$, we see that we may represent classes in $H^{1}\left(k^{\prime}, \mu_{m}\right)=k^{\prime \times} / k^{\prime \times m}$ by points in $\Omega_{1}$, and hence we conclude that $\chi$ is orthogonal to $H^{1}\left(k^{\prime}, \mu_{m}\right)$ for Tate's local duality pairing. This implies $\chi=0$, as desired.

b) We deduce via a restriction-corestriction argument from the case $T=\mathbf{G}_{m}$ that the groups $\amalg_{S}^{2}(T)$ and $\amalg_{\omega}^{2}(T)$ are of finite exponent. On the other hand, using the exact sequence

$$
0 \rightarrow \amalg^{2}(T) \rightarrow \amalg_{S}^{2}(T) \rightarrow \bigoplus_{v \in S} H^{2}\left(K_{v}, T\right),
$$

we obtain that $\amalg_{S}^{2}(T)$ is of cofinite type because $\amalg^{2}(T)$ is finite by Proposition 2.6 (2) and for each $n>0$ the Kummer sequence induces a surjection of the finite group $H^{1}\left(K_{v, n} T\right)$ onto ${ }_{n} H^{2}\left(K_{v}, T\right)$.

We now turn to statements concerning weak approximation. Given a variety $Y$ over $K$, we equip the sets $Y\left(K_{v}\right)$ with the $v$-adic topology defined by the discrete valuation coming from the closed point $v \in X$. By definition, weak approximation holds for $Y$ if the diagonal image of $Y(K)$ is dense in the topological direct product of the $Y\left(K_{v}\right)$. Note that this is the case when $Y$ is smooth and $K$-rational. Indeed, for the affine space weak approximation is just the classical Artin-Whaples approximation lemma ([21], Theorem XII.1.2) for discrete valuations, and the general case follows from the implicit function theorem for ultrametric valuations ([28), Part II, $\S I I I .10)$ which implies that for all $v$ and all nonempty Zariski open subsets $U \subset X$ the subset $U\left(K_{v}\right)$ is dense in $X\left(K_{v}\right)$ for the $v$-adic topology.

Theorem 3.3. Let $T$ be a torus over $K$.

a) For a finite set $S \subset X^{(1)}$ of places denote by $\overline{T(K)}_{S}$ be the closure of $T(K)$ in $\prod_{v \in S} T\left(K_{v}\right)$. There is an exact sequence of finite groups

$$
0 \rightarrow \overline{T(K)}_{S} \rightarrow \prod_{v \in S} T\left(K_{v}\right) \stackrel{\lambda_{S}}{\rightarrow} \amalg_{S}^{2}\left(T^{\prime}\right)^{D} \rightarrow \amalg^{1}(T) \rightarrow 0
$$

where the map $\lambda_{S}$ is defined using the local duality pairings $\langle,\rangle_{v}$ by

$$
\left(t_{v}\right) \mapsto\left(t^{\prime} \mapsto \sum_{v \in S}\left\langle t_{v}, t_{v}^{\prime}\right\rangle_{v}\right)
$$

for $t_{v} \in T\left(K_{v}\right)$ and $t^{\prime} \in \amalg_{S}^{2}\left(T^{\prime}\right)$.

b) Similarly, denote by $\overline{T(K)}$ the closure of $T(K)$ in the (topological) product of the $T\left(K_{v}\right)$ for all $v$. There is an exact sequence

$$
0 \rightarrow \overline{T(K)} \rightarrow \prod_{v \in X^{(1)}} T\left(K_{v}\right) \stackrel{\lambda_{\omega}}{\longrightarrow} \amalg_{\omega}^{2}\left(T^{\prime}\right)^{D} \rightarrow \amalg^{1}(T) \rightarrow 0
$$

with an analogously defined map $\lambda_{\omega}$. 
Note that the maps $\lambda_{S}$ and $\lambda_{\omega}$ are continuous because the local duality pairings $H^{0}\left(K_{v}, T\right) \rightarrow H^{2}\left(K_{v}, T^{\prime}\right)^{D}$ factor through the profinite completions $H^{0}\left(K_{v}, T\right)^{\wedge}$.

Proof. a) We follow the method of Sansuc 25. First, note that a quasi-trivial $K$-torus satisfies weak approximation as it is $K$-rational, and therefore the cokernel of the first map in (13) does not change if we multiply $T$ by a quasi-trivial torus. On the other hand, Lemma $3.2 a$ ) and Hilbert's Theorem 90 imply that the last two terms in the sequence do not change either when $T$ is multiplied by a quasi-trivial torus. So we are free to perform such an operation during the proof. Also, if the statement holds for some finite direct power $T^{m}$ of $T$, it holds for $T$ as well. So up to replacing $T$ by some $T^{m} \times Q$ with $Q$ quasi-trivial, we may apply a lemma of Ono (see e.g.[25], Lemma 1.7) to find an exact sequence of commutative $K$-group schemes

$$
0 \rightarrow F \rightarrow R \rightarrow T \rightarrow 0
$$

where $R$ is a quasi-trivial torus and $F$ is finite. This induces a dual exact sequence of Galois modules

$$
0 \rightarrow F^{\prime} \rightarrow T^{\prime} \rightarrow R^{\prime} \rightarrow 0
$$

where $F^{\prime}=\operatorname{Hom}(F, \mathbf{Q} / \mathbf{Z}(2))$ as usual. (Indeed, the map $T^{\prime} \rightarrow R^{\prime}$ is surjective since the map of cocharacters $\breve{R} \rightarrow \breve{T}$ is injective by finiteness of $F$. On the other hand, tensoring the exact sequence of character groups

$$
0 \rightarrow \widehat{T} \rightarrow \widehat{R} \rightarrow \operatorname{Hom}(F, \mathbf{Q} / \mathbf{Z}(1)) \rightarrow 0
$$

by $\mathbf{Z}(1)[1]$ in the derived sense we obtain an exact triangle

$$
\operatorname{Hom}(F, \mathbf{Q} / \mathbf{Z}(2)) \rightarrow \widehat{T}(1)[1] \rightarrow \widehat{R}(1)[1] \rightarrow \operatorname{Hom}(F, \mathbf{Q} / \mathbf{Z}(2))[1]
$$

in which using formula (3) we may identify the middle map with the surjection $T^{\prime} \rightarrow R^{\prime}$.) There is a commutative diagram with exact rows :

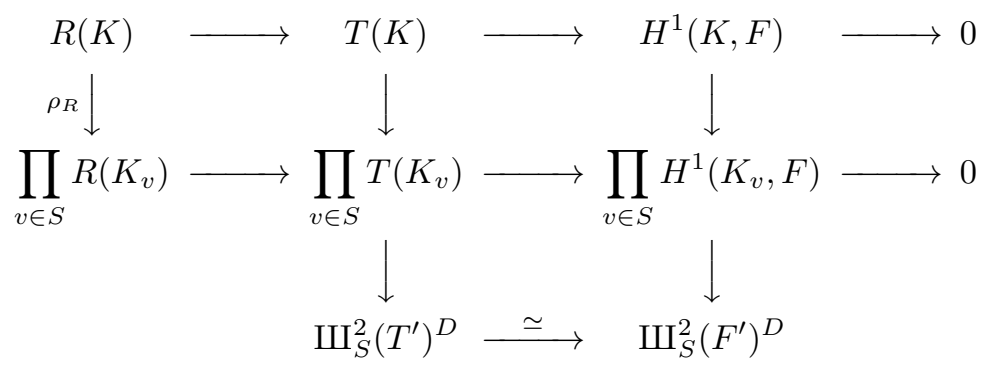

where the isomorphism at the bottom comes from the vanishing of the groups $H^{1}\left(K, R^{\prime}\right)$ and $\amalg_{S}^{2}\left(R^{\prime}\right)$ for the quasi-trivial torus $R^{\prime}$ (Lemma $\left.3.2 a\right)$ ).

The last column is exact by Lemma 3.1. Moreover, the map $\rho_{R}$ has dense image, again because $R$ is a quasi-trivial torus. A diagram chasing therefore yields an exact sequence

$$
0 \rightarrow \overline{T(K)}_{S} \rightarrow \prod_{v \in S} T\left(K_{v}\right) \rightarrow \amalg_{S}^{2}\left(T^{\prime}\right)^{D}
$$

To get the exactness of the last three terms in (13), we start from the exact sequence

$$
0 \rightarrow \amalg^{2}\left(T^{\prime}\right) \rightarrow \amalg_{S}^{2}\left(T^{\prime}\right) \rightarrow \bigoplus_{v \in S} H^{2}\left(K_{v}, T^{\prime}\right) .
$$

Dualizing this sequence, we get using Proposition 1.1 and Theorem 1.4 an exact sequence

$$
\prod_{v \in S} T\left(K_{v}\right)^{\wedge} \rightarrow \amalg_{S}^{2}\left(T^{\prime}\right)^{D} \rightarrow \amalg^{1}(T) \rightarrow 0 .
$$


But $\prod_{v \in S} T\left(K_{v}\right)$ and its profinite completion $\prod_{v \in S} T\left(K_{v}\right)^{\wedge}$ have the same image in $\amalg_{S}^{2}\left(T^{\prime}\right)^{D}$, because the latter is a finite group by Lemma $\left.3.2 b\right)$.

b) Taking the projective limit over $S$ in (13), we obtain the exactness of

$$
0 \rightarrow \overline{T(K)} \rightarrow \prod_{v \in X^{(1)}} T\left(K_{v}\right) \rightarrow \amalg_{\omega}^{2}\left(T^{\prime}\right)^{D}
$$

To get the exactness of the last three terms, we proceed as in $a$ ). After dualizing the exact sequence

$$
0 \rightarrow \amalg^{2}\left(T^{\prime}\right) \rightarrow \amalg_{\omega}^{2}\left(T^{\prime}\right) \rightarrow \bigoplus_{v \in X^{(1)}} H^{2}\left(K_{v}, T^{\prime}\right)
$$

we see that it is sufficient to prove that the image of $\prod_{v \in X^{(1)}} T\left(K_{v}\right)$ in $\amalg_{\omega}^{2}\left(T^{\prime}\right)^{D}$ is closed. It is therefore sufficient to show that the quotient of $\prod_{v \in X^{(1)}} T\left(K_{v}\right)$ by $\overline{T(K)}$ is compact. Using diagram (15), we obtain that this quotient is the same as the quotient of the profinite group $\prod_{v \in X^{(1)}} H^{1}\left(K_{v}, F\right)$ by the closure of the image of $H^{1}(K, F)$, hence it is compact as well. This concludes the proof.

Recall that $T$ is said to satisfy the weak weak approximation property if there exists a finite set $S_{0} \subset X^{(1)}$ such that for every finite set $S \subset X^{(1)}$ with $S \cap S_{0}=\emptyset$ the set $T(K)$ is dense in $\prod_{v \in S} T\left(K_{v}\right)$.

Corollary 3.4. A $K$-torus $T$ satisfies the weak weak approximation property if and only if $\amalg_{\omega}^{2}\left(T^{\prime}\right)$ is finite.

Proof. Assume first $\amalg_{\omega}^{2}\left(T^{\prime}\right)$ finite, and let $S_{0}$ be the union of all places $v$ such that there exists $\alpha \in \amalg_{\omega}^{2}\left(T^{\prime}\right)$ with $\alpha_{v} \neq 0$. Then $S_{0}$ is finite. If $S \subset X^{(1)}$ is a finite set with $S \cap S_{0}=\emptyset$, then by construction we have $\amalg_{S}^{2}\left(T^{\prime}\right)=\amalg^{2}\left(T^{\prime}\right)$, which implies that $T(K)$ is dense in $\prod_{v \in S} T\left(K_{v}\right)$ by Theorem $\left.3.3 a\right)$ and Theorem 1.4 .

Conversely, if there exists a finite set $S_{0} \subset X^{(1)}$ such that $T$ satisfies weak approximation outside $S_{0}$, then for every finite set of places $S$ disjoint from $S_{0}$ we have $\amalg_{S}^{2}\left(T^{\prime}\right)=\amalg^{2}\left(T^{\prime}\right)$ by Theorem $\left.3.3 a\right)$ and Theorem 1.4. Hence there is an exact sequence

$$
0 \rightarrow \amalg^{2}\left(T^{\prime}\right) \rightarrow \amalg_{\omega}^{2}\left(T^{\prime}\right) \rightarrow \bigoplus_{v \in S_{0}} H^{2}\left(K_{v}, T^{\prime}\right)
$$

Now the finiteness of $\amalg_{\omega}^{2}\left(T^{\prime}\right)$ follows in the same way as in the proof of Lemma 3.2 $b)$.

We close this section by observing that, in contrast to the number field case, it may happen that for a $K$-torus $T$ the group $\amalg_{\omega}^{2}(T)$ is infinite, and therefore the obstruction in Theorem 3.3 is not finite.

Proposition 3.5. The exists a torus $T$ over $K=\mathbf{Q}_{p}(t)$ with $\amalg_{\omega}^{2}(T)$ infinite.

Proof. It suffices to find a torus $Q$ over $K=\mathbf{Q}_{p}(t)$ with infinite $\amalg_{\omega}^{1}(Q)$. Indeed, since for a quasi-trivial torus $P$ we have $H^{1}(K, P)=\amalg_{\omega}^{2}(P)=0$, choosing a flasque resolution $1 \rightarrow T \rightarrow P \rightarrow Q \rightarrow 1$ yields $\amalg_{\omega}^{1}(Q) \cong \amalg_{\omega}^{2}(T)$.

One can then choose $Q$ to be the base change to $K$ of a coflasque $\mathbf{Q}_{p}$-torus $Q_{0}$. Since $Q_{0}$ is coflasque, we have by definition $H^{1}\left(k^{\prime}, \widehat{Q}_{0}\right)=0$ for every finite 
extension $k^{\prime} \mid \mathbf{Q}_{p}$, whence $H^{1}\left(k^{\prime}, Q_{0}\right)=0$ by Tate local duality for tori. This implies $H^{1}(K, Q)=\amalg_{\omega}^{1}(Q)$. Indeed, each class in $\alpha \in H^{1}(K, Q)$ comes from a class in $H^{1}(U, \mathcal{Q})$ for a suitable open subset $U \subset \mathbf{P}_{\mathbf{Q}_{p}}^{1}$ and model $\mathcal{Q}$ over $U$, and thus for $v \in U$ the image of $\alpha$ in $H^{1}\left(K_{v}, Q\right)$ comes from $H^{1}\left(\mathcal{O}_{v}, \mathcal{Q}\right) \cong H^{1}\left(k(v), Q_{0}\right)$ which is 0 by the above.

Choose moreover $Q_{0}$ in such a way that $H^{1}\left(\mathbf{Q}_{p}, \widehat{Q}_{0}^{\prime}\right) \neq 0$ for the dual flasque torus $Q_{0}^{\prime}$. For an explicit example, one may take $Q_{0}^{\prime}$ to be the flasque torus in a flasque resolution of $\mathbf{R}_{k^{\prime} \mid \mathbf{Q}_{p}}^{1} \mathbf{G}_{m}$, with $G:=\mathrm{Gal}\left(k^{\prime} \mid \mathbf{Q}_{p}\right)$ isomorphic to $(\mathbf{Z} / 2 \mathbf{Z})^{2}$. Indeed, we have $H^{1}\left(\mathbf{Q}_{p}, \widehat{Q}_{0}^{\prime}\right) \cong H^{3}(G, \mathbf{Z})$ by $([9]$, Proposition 7 , page 191 ; see also Lemma 5 and Proposition 6 of the same paper), but $H^{3}(G, \mathbf{Z}) \cong \mathbf{Z} / 2 \mathbf{Z}$ for our $G$ by ([27], I.4.4, Proposition 28).

Now fix a nonzero element $a \in H^{1}\left(\mathbf{Q}_{p}, \widehat{Q}_{0}^{\prime}\right) \neq 0$. For each $b \in \mathbf{Q}_{p}$ consider the cup-product $A_{b}:=i(a) \cup(t-b)$ in $H^{1}(K, Q)$, where $(t-b)$ is viewed as an element of $H^{0}\left(K, \mathbf{G}_{m}\right)$ and $i$ is the composite map $H^{1}\left(k(b), \widehat{Q}^{\prime}\right) \stackrel{\text { inf }}{\rightarrow} H^{1}\left(K_{b}, \widehat{Q}^{\prime}\right) \rightarrow$ $H^{1}\left(K, \widehat{Q}^{\prime}\right)$ (observe that $\widehat{Q}^{\prime} \otimes \mathbf{G}_{m}=Q$ ). The image of $A_{b}$ by the residue map $H^{1}(K, Q) \rightarrow H^{1}\left(k(b), \widehat{Q}^{\prime}\right)$ is $a$, so $A_{b}$ does not come from $H^{1}\left(U_{b}, \mathcal{Q}\right)$ for any open neighbourhood $U_{b}$ of $b$ (see the remark below for the residue argument used here). Since $\mathbf{Q}_{p}$-rational points are Zariski dense in $\mathbf{P}_{\mathbf{Q}_{p}}^{1}$, there is no Zariski open subset $U \subset \mathbf{P}_{\mathbf{Q}_{p}}^{1}$ such that every element of $H^{1}(K, Q)$ comes from $H^{1}(U, \mathcal{Q})$. Therefore $H^{1}(K, Q)$ must be infinite.

Remark 3.6. Some explanation is in order concerning the residue maps used in the above proof. They appear in various guises in the literature, but the following simple direct approach which is well adapted for our purposes is perhaps worth noting. Given a complete discrete valuation ring $\mathcal{O}_{v}$ with closed point $v$, fraction field $K_{v}$ and perfect residue field $k(v)$, it is shown in [23], II.1.7(b) that there are isomorphisms $H_{v}^{i+1}\left(\mathcal{O}_{v}, \mathbf{G}_{m}\right) \stackrel{\sim}{\rightarrow} H^{i}(k(v), \mathbf{Z})$ for $i \geq 1$, basically induced by the valuation map. Given an $\mathcal{O}_{v}$-torus $\mathcal{T}$, tensoring $\mathbf{G}_{m}$ with $\check{\mathcal{T}}=\widehat{\mathcal{T}}^{\prime}$ we may repeat the construction in the reference to obtain an isomorphism $H_{v}^{i+1}\left(\mathcal{O}_{v}, T\right) \stackrel{\sim}{\rightarrow} H^{i}(k(v), \check{T})$. On the other hand, localization on $\operatorname{Spec} \mathcal{O}_{v}$ yields an exact sequence

$$
H^{i}\left(\mathcal{O}_{v}, \mathcal{T}\right) \rightarrow H^{i}\left(K_{v}, T\right) \rightarrow H_{v}^{i+1}\left(\mathcal{O}_{v}, \mathcal{T}\right)
$$

whence the residue map $H^{i}\left(K_{v}, T\right) \rightarrow H^{i}(k(v), \breve{T})$ used above for $i=1$. By construction, for $i=1$ its value is $a$ on elements of the form $\inf (a) \cup \pi$ for $a \in$ $H^{1}(k(v), \breve{T})$ and $\pi$ a uniformizer, and it vanishes on classes coming from $H^{1}\left(\mathcal{O}_{v}, \mathcal{T}\right)$.

In the above proof this construction was applied to completions of local rings of smooth $k$-curves. In fact, it can be shown using the global localization sequence in étale cohomology that on a smooth $k$-curve $U$ with fraction field $K$ the classes in $H^{i}(K, T)$ that come from some class in $H^{i}(U, \mathcal{T})$ are exactly those whose residues are trivial at all closed points of $U$, but we did not need this fact.

\section{Reciprocity obstructions to WEAK APPROXimation}

For varieties over number fields, unramified elements in the Brauer group have been used for a long time to construct obstructions to weak approximation. As was observed by Colliot-Thélène in the 1990's, such obstructions can be defined using higher unramified cohomology groups as well. A detailed exposition is given in $\S 2$ of the recent preprint [8]; we give here a reasonably self-contained exposition of the special case we need.

Recall that given a field $F$ and a smooth $F$-variety $Y$ with function field $F(Y)$, the unramified part $H_{\mathrm{nr}}^{i}\left(F(Y), \mu_{n}^{\otimes j}\right)$ of the cohomology group $H^{i}\left(F(Y), \mu_{n}^{\otimes j}\right)$ is 
defined as the group of cohomology classes that come from $H^{i}\left(A, \mu_{n}^{\otimes j}\right)$ for every discrete valuation ring $A \supset F$ with fraction field $F(Y)$. If moreover $Y$ is proper over $F$, the Bloch-Ogus theorem for the local rings of $Y$ implies that $H_{\mathrm{nr}}^{i}\left(F(Y), \mu_{n}^{\otimes j}\right)$ consists of the classes that come from $H^{i}\left(\mathcal{O}_{Y, P}, \mu_{n}^{\otimes j}\right)$ for every point $P \in Y$ (see 4, Theorem 4.1.1). As pointed out on p. 156 of [5], for an overfield $F^{\prime} \supset F$ this enables one to define an evaluation pairing

$$
Y\left(F^{\prime}\right) \times H_{\mathrm{nr}}^{i}\left(F(Y), \mu_{n}^{\otimes j}\right) \rightarrow H^{i}\left(F^{\prime}, \mu_{n}^{\otimes j}\right)
$$

as follows: given a point $M: \operatorname{Spec} F^{\prime} \rightarrow Y$ whose image is the point $P \in Y$, one evaluates $\alpha \in H_{\mathrm{nr}}^{i}\left(F(Y), \mu_{n}^{\otimes j}\right)$ at $M$ by lifting it (uniquely) to $H^{i}\left(\mathcal{O}_{X, P}, \mu_{n}^{\otimes j}\right)$ and then taking its image by the map $H^{i}\left(\mathcal{O}_{X, P}, \mu_{n}^{\otimes j}\right) \rightarrow H^{i}\left(F^{\prime}, \mu_{n}^{\otimes j}\right)$ induced by $M$. Note that this definition also works for $Y$ not necessarily proper but having a smooth compactification over $F$.

If now $F=K$ is the function field of a curve $X$ over a finite extension $k \mid \mathbf{Q}_{p}$ and $F^{\prime}=K_{v}$ is the completion of $K$ at a closed point $v \in X$, for $i=3$ and $j=2$ the above construction yields a pairing

$$
Y\left(K_{v}\right) \times H_{\mathrm{nr}}^{3}\left(K(Y), \mu_{n}^{\otimes 2}\right) \rightarrow H^{3}\left(K_{v}, \mu_{n}^{\otimes 2}\right) .
$$

¿From this we can construct a pairing

$$
\prod_{v \in X^{(1)}} Y\left(K_{v}\right) \times H_{\mathrm{nr}}^{3}(K(Y), \mathbf{Q} / \mathbf{Z}(2)) \rightarrow \mathbf{Q} / \mathbf{Z}
$$

using that $H^{3}\left(K_{v}, \mathbf{Q} / \mathbf{Z}(2)\right) \cong \mathbf{Q} / \mathbf{Z}$ for all $v$, as recalled in Section 1. To see that it is well defined, we show:

Lemma 4.1. For fixed $\alpha \in H_{\mathrm{nr}}^{3}\left(K(Y), \mu_{n}^{\otimes 2}\right)$ the map $Y\left(K_{v}\right) \rightarrow \mathbf{Q} / \mathbf{Z}$ induced by evaluating $\alpha$ via (16) is 0 for all but finitely many $v$.

Proof. This is part of (8], Proposition 2.5). The argument is the following. Choosing a model $\mathcal{Y}^{c} \rightarrow U$ of a smooth compactification $Y^{c}$ over a sufficiently small open $U \subset X$, the element $\alpha$ lies in $H^{3}\left(\mathcal{O}_{\mathcal{Y}^{c}, Q}, \mathbf{Q} / \mathbf{Z}(2)\right)$ for all but finitely many codimension 1 points $Q$ of the $k$-variety $\mathcal{Y}^{c}$. By the assumption on $\alpha$, the exceptional $Q$ lie in finitely many closed fibres $\mathcal{Y}_{v_{1}}^{c}, \ldots, \mathcal{Y}_{v_{r}}^{c}$ of $\mathcal{Y}^{c} \rightarrow U$. Therefore for $v \neq v_{i}$ we have $\alpha \in H^{3}\left(\mathcal{O}_{\mathcal{Y}^{c}, P}, \mathbf{Q} / \mathbf{Z}(2)\right)$ for all points $P \in \mathcal{Y}_{v}^{c}$ and hence the map $Y^{c}\left(K_{v}\right)=\mathcal{Y}^{c}\left(\mathcal{O}_{v}\right) \rightarrow \mathbf{Z} / n \mathbf{Z}$ induced by $\alpha$ factors through $H^{3}\left(\mathcal{O}_{v}, \mathbf{Q} / \mathbf{Z}(2)\right)=0$.

By Saito ([24], Proof of Proposition II.1.2), the sequence

$$
H^{3}(K, \mathbf{Q} / \mathbf{Z}(2)) \rightarrow \bigoplus_{v \in X^{(1)}} H^{3}\left(K_{v}, \mathbf{Q} / \mathbf{Z}(2)\right) \stackrel{\Sigma}{\longrightarrow} \mathbf{Q} / \mathbf{Z}
$$

is a complex. This also follows from the generalized Weil reciprocity law ([27], Chapter II, Annexe, §3) which is valid over an arbitrary field. Therefore the pairing (17) annihilates the diagonal image of $Y(K)$ by a reciprocity law and also its closure in the product topology by a continuity argument combined with Lemma 4.1. This gives rise to the obstruction to weak approximation defined by elements in $H_{\mathrm{nr}}^{3}(K(Y), \mathbf{Q} / \mathbf{Z}(2))$.

Of course, subgroups of $H_{\mathrm{nr}}^{3}(K(Y), \mathbf{Q} / \mathbf{Z}(2))$ define finer obstructions to weak approximation. The following theorem identifies such a subgroup in the case of tori which in fact constitutes the only obstruction.

Theorem 4.2. Let $T$ be a K-torus. There is a homomorphism

$$
u: \amalg_{\omega}^{2}\left(T^{\prime}\right) \rightarrow H_{\mathrm{nr}}^{3}(K(T), \mathbf{Q} / \mathbf{Z}(2))
$$

such that each system $\left(P_{v}\right)$ of local points of $T$ annihilated by $\operatorname{Im}(u)$ under the pairing (17) is in the closure of the diagonal image of $T(K)$ for the product topology. 
Proof. Take a flasque resolution

$$
1 \rightarrow S \rightarrow R \rightarrow T \rightarrow 1
$$

of $T$ as in (1). It allows one to consider $R$ as $T$-torsor under $S$, with cohomology class $[R] \in H^{1}(T, S)$. Choose a smooth compactification $T^{c}$ of $T$. Since $S$ is flasque, by ([10, Theorem 2.2) the $T$-torsor $R$ extends to a $T^{c}$-torsor $Y \rightarrow T^{c}$ under $S$ with associated cohomology class $[Y] \in H^{1}\left(T^{c}, S\right)$. The pairing $S \otimes S^{\prime} \rightarrow \mathbf{Z}(2)[2]$ between $S$ and the dual torus $S^{\prime}$ defined in Section 1 induces a homomorphism

$$
H^{1}\left(K, S^{\prime}\right) \rightarrow H^{4}\left(T^{c}, \mathbf{Z}(2)\right)
$$

given by $a \mapsto a_{T^{c}} \cup[Y]$.

Next recall (e.g. from [19], Prop. 2.9) that there is a natural map

$$
H^{4}\left(T^{c}, \mathbf{Z}(2)\right) \rightarrow H_{\mathrm{nr}}^{3}(K(T), \mathbf{Q} / \mathbf{Z}(2))
$$

defined as follows. The Leray spectral sequence for the change-of-sites map $\alpha$ : $T_{\text {et }}^{c} \rightarrow T_{\text {Zar }}^{c}$ yields an edge map

$$
H^{4}\left(T^{c}, \mathbf{Z}(2)\right) \rightarrow H_{\mathrm{Zar}}^{0}\left(T^{c}, \mathbf{R}^{4} \alpha_{*} \mathbf{Z}(2)\right) .
$$

According to ([19], Theorem 2.6. c)) the natural map $\mathbf{Q}(2)_{\mathrm{Zar}} \rightarrow \mathbf{R} \alpha_{*} \mathbf{Q}(2)$ is an isomorphism in the derived category of Zariski sheaves. But since $\mathbf{Q}(2)_{\text {Zar }}$ is concentrated in degrees $\leq 2$, we have $\mathbf{R}^{3} \alpha_{*} \mathbf{Q}(2)=\mathbf{R}^{4} \alpha_{*} \mathbf{Q}(2)=0$ and therefore the exact sequence

$$
0 \rightarrow \mathbf{Z}(i) \rightarrow \mathbf{Q}(i) \rightarrow \mathbf{Q} / \mathbf{Z}(i) \rightarrow 0
$$

induces an isomorphism $\mathbf{R}^{4} \alpha_{*} \mathbf{Z}(2) \cong \mathbf{R}^{3} \alpha_{*} \mathbf{Q} / \mathbf{Z}(2)$. Finally, we have an isomorphism

$$
H_{\mathrm{Zar}}^{0}\left(T^{c}, \mathbf{R}^{3} \alpha_{*} \mathbf{Q} / \mathbf{Z}(2)\right) \cong H_{\mathrm{nr}}^{3}(K(T), \mathbf{Q} / \mathbf{Z}(2))
$$

by the Gersten resolution (see [4, Theorem 4.1.1).

Observe that although $H^{4}\left(T^{c}, \mathbf{Z}(2)\right)$ and $H^{3}\left(T^{c}, \mathbf{Q} / \mathbf{Z}(2)\right)$ are not isomorphic in general, there is an isomorphism between $H^{4}\left(K\left(T^{c}\right), \mathbf{Z}(2)\right)$ and $H^{3}\left(K\left(T^{c}\right), \mathbf{Q} / \mathbf{Z}(2)\right)$ by a similar argument as above. Moreover, the construction of the Gersten resolution implies that the restriction map $H^{4}\left(T^{c}, \mathbf{Z}(2)\right) \rightarrow H^{4}(K(T), \mathbf{Z}(2))$ and the map (19) fit in a commutative diagram

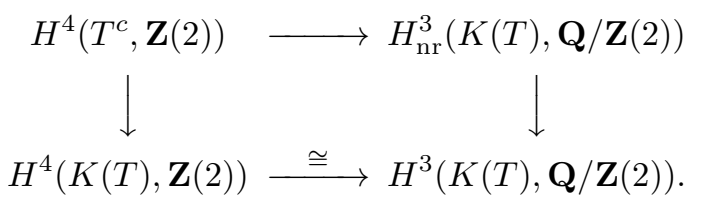

Now consider the dual exact sequence

$$
1 \rightarrow T^{\prime} \rightarrow R^{\prime} \rightarrow S^{\prime} \rightarrow 1
$$

coming from the flasque resolution. It induces the exact sequence

$$
0 \rightarrow H^{1}\left(K, S^{\prime}\right) \rightarrow H^{2}\left(K, T^{\prime}\right) \rightarrow H^{2}\left(K, R^{\prime}\right),
$$

and since $\amalg_{\omega}^{2}\left(R^{\prime}\right)=0$ by Lemma 3.2 (the dual of a quasi-trivial torus being quasitrivial), we obtain an isomorphism $\amalg_{\omega}^{1}\left(S^{\prime}\right) \stackrel{\sim}{\rightarrow} \amalg_{\omega}^{2}\left(T^{\prime}\right)$.

All in all, following the composite map

$$
\amalg_{\omega}^{2}\left(T^{\prime}\right) \stackrel{\sim}{\rightarrow} \amalg_{\omega}^{1}\left(S^{\prime}\right) \hookrightarrow H^{1}\left(K, S^{\prime}\right)
$$

by the composition of the maps (18) and (19) we obtain a homomorphism $u$ : $\amalg_{\omega}^{2}\left(T^{\prime}\right) \rightarrow H_{\mathrm{nr}}^{3}(K(T), \mathbf{Q} / \mathbf{Z}(2))$ as in the statement of the theorem.

Let $\left(P_{v}\right) \in \prod_{v \in X^{(1)}} T\left(K_{v}\right)$. Observe that the evaluation

$$
P_{v} \mapsto[Y]\left(P_{v}\right)=[R]\left(P_{v}\right) \in H^{1}\left(K_{v}, S\right)
$$


is given by the coboundary map $\partial_{v}: T\left(K_{v}\right) \rightarrow H^{1}\left(K_{v}, S\right)$ corresponding to the flasque resolution (11). Consider the commutative diagram with exact rows

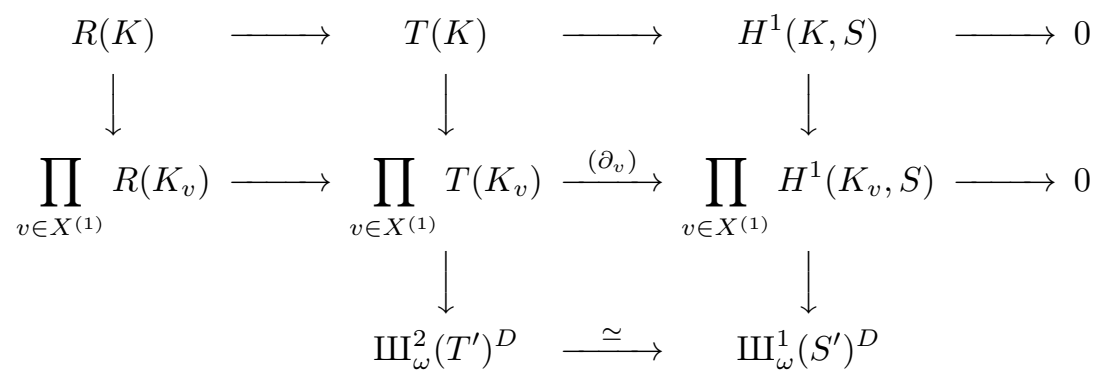

in which the vertical maps are induced by the local duality pairings and the horizontal ones by the long exact sequence coming from the flasque resolution. Since $R$ is quasi-trivial, the upper left vertical map has dense image. Hence the diagram together with Theorem $3.3 b$ ) shows that $\left(P_{v}\right)$ is in the closure of $T(K)$ if and only if $\left(\partial_{v}\left(P_{v}\right)\right)$ is orthogonal to $\amalg_{\omega}^{1}\left(S^{\prime}\right)$, which means

$$
\left.0=\sum_{v \in X^{(1)}}\left\langle a_{v}, \partial_{v}\left(P_{v}\right)\right\rangle_{v}=\sum_{v \in X^{(1)}} a_{v} \cup \partial_{v}\left(P_{v}\right)=\sum_{v \in X^{(1)}}\left(a_{T^{c}} \cup[Y]\right)\left(P_{v}\right)\right)
$$

for every $a \in \amalg_{\omega}^{1}\left(S^{\prime}\right)$. Here $\left(a_{T^{c}} \cup[Y]\right)\left(P_{v}\right)$ denotes the image of the class $\left(a_{T^{c}} \cup[Y]\right) \in H^{4}\left(T^{c}, \mathbf{Z}(2)\right)$ by the evaluation map $H^{4}\left(T^{c}, \mathbf{Z}(2)\right) \rightarrow H^{4}\left(K_{v}, \mathbf{Z}(2)\right) \cong$ $\mathbf{Q} / \mathbf{Z}$ associated with $P_{v}$; it is 0 for all but finitely many $v$ as $a \in \amalg_{\omega}^{1}\left(S^{\prime}\right)$. Finally, diagram (20) shows that the condition

$$
\left.\sum_{v \in X^{(1)}}\left(a_{T^{c}} \cup[Y]\right)\left(P_{v}\right)\right)=0
$$

means precisely that $\left(P_{v}\right)$ is orthogonal to the image of $u$ for the pairing (17).

\section{Remarks 4.3.}

1. In their recent work [1], Blinstein and Merkurjev also construct a homomorphism

$$
H^{1}\left(K, S^{\prime}\right) \rightarrow H_{\mathrm{nr}}^{3}(K(T), \mathbf{Q} / \mathbf{Z}(2))
$$

as a consequence of their Theorems 4.4 and 5.6. By comparing the two constructions (and taking in particular the proof of Proposition 5.3 of [1 into account), one sees that the map of Blinstein and Merkurjev is none but the composite of our maps (18) and (19).

2. One can also construct the map (21) at a finite level, avoiding the use of the complex $\mathbf{Z}(2)$. Namely, we can find an integer $n>0$ annihilating the group $H^{1}\left(K, S^{\prime}\right)$ (for example, the degree of an extension splitting $S$ ). We can then send $[Y] \in H^{1}\left(T^{c}, S\right)$ to a class in $H^{2}\left(T^{c},{ }_{n} S\right)$ by a coboundary map $\partial_{n}$ and lift $a \in H^{1}\left(K, S^{\prime}\right)$ to $a_{n} \in H^{1}\left(K,{ }_{n} S^{\prime}\right)$. The cup-product $\left(a_{n}\right)_{T^{c}} \cup \partial_{n}([Y])$ induced by the pairing ${ }_{n} S^{\prime} \otimes{ }_{n} S \rightarrow \mu_{n}^{\otimes 2}$ lives in $H^{3}\left(T^{c}, \mu_{n}^{\otimes 2}\right)$ and restricts to a class in $H_{\mathrm{nr}}^{3}\left(K(T), \mu_{n}^{\otimes 2}\right)$ via an argument involving the Gersten resolution as in the construction of the map (19). One can check that this construction is independent of the choices made and yields the same map $H^{1}\left(K, S^{\prime}\right) \rightarrow H_{\mathrm{nr}}^{3}(K(T), \mathbf{Q} / \mathbf{Z}(2))$ as above.

3. In the classical case where $T$ is defined not over $K$ but over a number field $k$, we have an isomorphism of cohomology groups

$$
H_{\mathrm{nr}}^{2}(k(T), \mathbf{Q} / \mathbf{Z}(1)) \cong H^{2}\left(T^{c}, \mathbf{Q} / \mathbf{Z}(1)\right)
$$

(see e.g. 4, Theorem 4.2.3). Therefore the evaluation pairing can be defined directly by evaluating classes in $H^{2}\left(T^{c}, \mathbf{Q} / \mathbf{Z}(1)\right)$, without passing through a local ring. In the above proof we have used an intermediate evaluation pairing with the 
group $H^{4}\left(T^{c}, \mathbf{Z}(2)\right)$ and then composed with the map (19) which is in fact surjective but not injective in general ([19], Proposition 2.9).

4. Using the same tools as in Section 6 of [15, one can show that the reciprocity obstruction to weak approximation associated with $H_{\mathrm{nr}}^{3}(K(G), \mathbf{Q} / \mathbf{Z}(2))$ is still the only one for a quasi-split reductive $K$-group $G$ such that the universal cover $G^{\text {sc }}$ of its derived subgroup satisfies weak approximation. This is in particular the case when $G^{\mathrm{sc}}$ is $K$-rational, e.g. $G^{\mathrm{sc}}=\mathrm{SL}_{n}$. The proof requires a non-commutative generalization of Theorem 3.3 which can be obtained by adapting the methods of [25] in their full generality. Also, one has to use the noncommutative flasque resolutions of 6 to prove a non-commutative generalization of Theorem 4.2 .

\section{REFERENCES}

[1] S. Blinstein, A. Merkurjev, Cohomological invariants of algebraic tori, preprint 2012, to appear in Algebra \&s Number Theory.

[2] S. Bloch, Algebraic cycles and higher K-theory, Adv. in Math. 61 (1986), 267-304.

[3] M. Borovoi, C. Demarche, Manin obstruction to strong approximation for homogeneous spaces, Comment. Math. Helv. 88 (2013), 1-54.

[4] J-L. Colliot-Thélène, Birational invariants, purity and the Gersten conjecture, in W. Jacob and A. Rosenberg (eds.) K-Theory and Algebraic Geometry: Connections with Quadratic Forms and Division Algebras, Proc. Symp. Pure Math. vol. 58 (1995), Part I, 1-64.

[5] Groupes linéaires sur les corps de fonctions de courbes réelles, J. reine angew. Math. 474 (1996) 139-167.

[6] Résolutions flasques des groupes linéaires connexes, J. reine angew. Math. 618 (2008), 77-133.

[7] J-L. Colliot-Thélène, R. Parimala, V. Suresh, Patching and local-global principles for homogeneous spaces over function fields of p-adic curves, Comment. Math. Helv. 87 (2012), 1011-1033.

[8] - Lois de réciprocité supérieures et points rationnels, preprint arXiv:1302.2377, 2013.

[9] J-L. Colliot-Thélène, J-J. Sansuc, La R-équivalence sur les tores, Ann. Sci. École Norm. Sup. 10 (1977), 175-229.

[10] , Principal homogeneous spaces under flasque tori; applications, Journal of Algebra 106 (1987), 148-205.

[11] T. Geisser, Duality via cycle complexes, Ann. of Math. 172 (2010), 1095-1126.

[12] T. Geisser, M. Levine, The Bloch-Kato conjecture and a theorem of Suslin-Voevodsky, J. reine angew. Math. 530 (2001), 55-103.

[13] M. J. Greenberg, Rational points in Henselian discrete valuation rings, Inst. Hautes Études Sci. Publ. Math. 31 (1966), 59-64.

[14] D. Harari, T. Szamuely, Arithmetic duality theorems for 1-motives, J. reine angew. Math. $\mathbf{5 7 8}$ (2005), 93-128.

[15] L L L L L L 2013.

[16] D. Harbater, J. Hartmann and D. Krashen, Local-global principles for torsors over arithmetic curves, preprint, 2011.

[17] G. Harder, Halbeinfache Gruppenschemata über Dedekindringen, Invent. Math. 4 (1968), 165-191.

[18] E. Hewitt, K. Ross, Abstract harmonic analysis I, Springer-Verlag, Berlin, 1963.

[19] B. Kahn, Classes de cycles motiviques étales, Algebra $\&$ Number Theory 6-7 (2012), 13691407.

[20] K. Kato, A generalization of local class field theory by using K-groups II, J. Fac. Sci. Univ. Tokyo 27 (1980), 603-683.

[21] Serge Lang, Algebra, third edition, Addison-Wesley, Reading, 1993.

[22] S. Lichtenbaum, Duality theorems for curves over p-adic fields, Invent. Math. 7 (1969), 120136.

[23] J.S. Milne, Arithmetic Duality Theorems, Academic Press, 1986.

[24] S. Saito, Class field theory for curves over local fields, J. Number Theory 21 (1985), 44-80.

[25] J-J. Sansuc, Groupe de Brauer et arithmétique des groupes algébriques linéaires sur un corps de nombres, J. reine angew. Math. 327 (1981), 12-80.

[26] C. Scheiderer, J. van Hamel, Cohomology of tori over p-adic curves, Math. Ann. 326 (2003), $155-183$. 
[27] J-P. Serre, Cohomologie Galoisienne (cinquième édition, révisée et complétée), Lecture Notes in Math. 5, Springer Verlag, 1994.

[28] _ Lie algebras and Lie groups (Second edition), Lecture Notes in Math. 1500, SpringerVerlag, Berlin, 1992.

[29] A. Suslin, V. Voevodsky, Bloch-Kato conjecture and motivic cohomology with finite coefficients, in The arithmetic and geometry of algebraic cycles, NATO Sci. Ser. C Math. Phys. Sci., vol. 548, Kluwer, 2000, 117-189.

[30] V. E. Voskresenskiǔ, Birational properties of linear algebraic groups (Russian), Izv. Akad. Nauk SSSR Ser. Mat. 34 (1970) 3-19.

Université de Paris-Sud Mathématique, BÂtiment 425, 91405 Orsay, France

E-mail address: David.Harari@math.u-psud.fr

Fachbereich Mathematik and Statistik, Universität Konstanz, D-78457 Konstanz, GERMANY

E-mail address: claus.scheiderer@uni-konstanz.de

Alfréd Rényi Institute of Mathematics, Hungarian Academy of Sciences, Reáltanoda utca 13-15, H-1053 Budapest, Hungary and Central European University, Nádor utca 9, H-1051 Budapest, Hungary

E-mail address: szamuely.tamas@renyi.mta.hu 